\title{
Infinite paths in planar graphs III, 1-way infinite paths
}

\author{
Xingxing $\mathrm{Yu}^{*}$ \\ School of Mathematics \\ Georgia Institute of Technology \\ Atlanta, GA 30332, USA \\ and \\ Center for Combinatorics \\ Nankai University \\ Tianjin, 300071, China
}

May 11, 2004

\begin{abstract}
An infinite graph is 2-indivisible if the deletion of any finite set of vertices from the graph results in exactly one infinite component. Let $G$ be a 4 -connected, 2indivisible, infinite, plane graph. It is known that $G$ contains a spanning 1-way infinite path. In this paper, we prove a stronger result by showing that, for any vertex $x$ and any edge $e$ on a facial cycle of $G$, there is a spanning 1-way infinite path in $G$ from $x$ and through $e$. Results will be used to establish a conjecture of Nash-Williams.
\end{abstract}

* Partially supported by NSF grant DMS-0245530 and NSA grant MDA-904-03-1-0052 


\section{Introduction and notation}

Notation and terminology not defined in this paper may be found in [9] and [10]. In 1931, Whitney [8] proved that every 4-connected planar triangulation contains a Hamilton cycle. Later, Tutte [7] proved that every 4-connected planar graph contains a Hamilton cycle. A natural extension of this theorem to infinite planar graphs is the existence of spanning 1-way infinite paths or 2-way infinite paths. This lead Nash-Williams to the following concept: A graph $G$ is $k$-indivisible, where $k$ is a positive integer, if, for any finite $X \subseteq V(G), G-X$ has at most $k-1$ infinite components. Nash-Williams ([2], [3], also see [5]) conjectured that a 4-connected, infinite, planar graph $G$ contains a spanning 1-way infinite path if, and only if, $G$ is 2-indivisible. This conjecture has been verified by Dean, Thomas and Yu [1]. Nash-Williams ([2] and [3]) also conjectured that a 4-connected, infinite, planar graph contains a spanning 2-way infinite path if, and only if, it is 3-indivisible. This conjecture is verified for 2-indivisible graphs in [9] and [10]. In order to establish this conjecture completely, we need results that are stronger than those in [1]. In particular, we need to prove the existence of a certain type of 1-way infinite paths in 2-indivisible graphs. For simplicity, we first state a consequence of our main result.

(1.1) Theorem. Let $G$ be a 4-connected, 2-indivisible, infinite, plane graph. Let $C$ be a facial cycle of $G, x \in V(C)$, and $e \in E(C)$. Then $G$ contains a spanning 1-way infinite path from $x$ and through $e$.

To state the main result of this paper, we recall the definition of a Tutte subgraph. Let $G$ be a graph (finite or infinite) and $P$ be a subgraph (finite or infinite) of $G$. A $P$-bridge of $G$ is a subgraph (finite or infinite) of $G$ which is induced by either (1) a single edge in $E(G)-E(P)$ with both incident vertices in $V(P)$ or $(2)$ the edges contained in a component $D$ of $G-V(P)$ and the edges from $D$ to $P$. (For any $X \subseteq V(G)$, we view $X$ as a subgraph of $G$ with $V(X)=X$ and $E(X)=\emptyset$, and hence, we can speak of $X$-bridges of $G$.) A $P$-bridge satisfying (2) is said to be nontrivial. If $B$ is a $P$-bridge of $G$, then the vertices in $V(P) \cap V(B)$ are called the attachments of $B$ (on $P$ ). We say that $P$ is a Tutte subgraph of $G$ if every $P$-bridge of $G$ is finite and has at most three attachments. For any subgraph $C$ in $G$, we say that $P$ is a $C$-Tutte subgraph in $G$ if $P$ is a Tutte subgraph of $G$ and every $P$-bridge of $G$ containing an edge of $C$ has at most two attachments.

Let $G$ be a graph and $C$ a subgraph of $G$; we say that $G$ is $(4, C)$-connected if, for any cut set $S$ of $G$ with $|S| \leq 3$, every component of $G-S$ contains a vertex of $C$. For vertices $x, v$ on a path $P$, we use $x P v$ denote the subpath of $P$ between $x$ and $v$. We can now state the main result of this paper. 
(1.2) Theorem. Let $G$ be a 2-connected, 2-indivisible, infinite plane graph, let $C$ be a facial cycle of $G$, let $x \in V(C)$ and $u v \in E(C)$ with $v \neq x$, and let $Q$ denote the subpath of $C-v$ between $u$ and $x$. Assume that $G$ is $(4, C)$-connected and $v$ is contained in the infinite component of $G-V(Q)$. Then $G$ contains a 1-way infinite $C$-Tutte path $P$ from $x$ such that $u v \in E(P)$ and $u \in V(x P v)$.

This paper is organized as follows. In Section 2, we briefly review the definition of a net and a structural result of 2-indivisible infinite plane graphs. We will prove, in Section 3 , several lemmas for extending Tutte paths in 2-connected graphs. In Section 4, we will prove a special case of (1.1), which will then be used in Section 5 as an induction basis to complete the proof of (1.1).

We consider simple graphs only. Let $G$ be a plane graph. The subgraph of $G$ consisting of vertices and edges incident with the infinite face is denoted by $\partial G$. Given any cycle $C$ in $G$ and given distinct $x, y \in V(C)$, we use $x C y$ to denote the clockwise segment of $C$ from $x$ to $y$ (which is a path). To avoid confusion, we adopt the convention that a graph is finite, unless mentioned otherwise.

\section{Nets}

For convenience, we recall from [10] the notation and definition of a net. By the Jordan curve theorem, any cycle $C$ in an infinite plane graph $G$ divides the plane into two closed regions (whose intersection is $C$ ). If exactly one of these two closed regions, say $\mathcal{R}$, contains only finitely many vertices and edges of $G$, then we use $I_{G}(C)$ to denote the subgraph of $G$ consisting of vertices and edges of $G$ contained in $\mathcal{R}$. Hence, $I_{G}(C)$ is a finite graph. When there is no danger of confusion, we use $I(C)$ instead of $I_{G}(C)$. Note that $C \subseteq I(C)$, and if $I(C)=C$ then $C$ is a facial cycle of $G$.

A net in an infinite plane graph $G$ is a sequence $N=\left(C_{1}, C_{2}, \cdots\right)$ of cycles in $G$ such that $I\left(C_{i}\right)$ is defined for all $i \geq 1$, and the following properties are satisfied:

(1) $I\left(C_{i}\right) \subseteq I\left(C_{i+1}\right)$ for all $i \geq 1$,

(2) $\bigcup_{i=1}^{\infty} I\left(C_{i}\right)=G$, and

(3) either $C_{i} \cap C_{j}=\emptyset$ for all $i \neq j$, or, for all $i \geq 1, C_{i} \cap C_{i+1}$ is a non-trivial path, $C_{i} \cap C_{i+1} \subseteq C_{i+1} \cap C_{i+2}$, and neither endvertex of $C_{i} \cap C_{i+1}$ is an endvertex of $C_{i+1} \cap C_{i+2}$.

If $C_{i} \cap C_{j}=\emptyset$ for all $i \neq j$, then $N$ is a radial net; otherwise, $N$ is a ladder net. Let $\partial N=\emptyset$ if $N$ is a radial net; otherwise, let $\partial N=\bigcup_{i=1}^{\infty}\left(C_{i} \cap C_{i+1}\right)$.

Note that from (2) and (3) that if an infinite plane graph has a net, then it is locally finite, that is, every vertex has finite degree. Also note from (3) that if $N$ is a ladder net in an infinite plane graph, then $\partial N$ is a 2-way infinite path. 
The following result is Theorem (2.4) in [10], which describes the structure of a 4-connected, 2-indivisible, infinite, plane graph.

(2.1) Theorem. Let $G$ be a 4-connected, 2-indivisible, infinite, plane graph, let $C$ be a facial cycle of $G$, and let $S$ denote the set of vertices of infinite degree in $G$. Then $|S| \leq 2$, and there is a set $F \subseteq E(G)$ such that

(1) for any $f \in F, f$ is incident with exactly one vertex in $S$,

(2) $G-F$ has a net $N=\left(C_{1}, C_{2}, \cdots\right), C \subseteq I\left(C_{1}\right), S \subseteq \partial N$, and, for any $f \in F$, both incident vertices of $f$ are contained in a common infinite $S$-bridge of $\partial N$,

(3) if $|S|=1$, then either one $S$-bridge of $\partial N$ contains all vertices incident with edges in $F$ or each $S$-bridge of $\partial N$ contains infinitely many vertices incident with edges in $F$, and

(4) if $|S|=2$, then, for any $T \subseteq V(G)-S$ with $|T| \leq 3, S$ is contained in a component of $(G-F)-T$.

It will be convenient to deal with certain embeddings of an infinite plane graph. An infinite plane graph $G$ is nicely embedded (or is a nice embedding) if, for every cycle $C$ in $G$ for which $I_{G}(C)$ is defined, $I_{G}(C)$ is contained in the closed disc bounded by $C$. The following result is Lemma (2.1) in [10].

(2.2) Lemma. If $G$ is a plane graph with a net and $C$ is a facial cycle of $G$, then $G$ has a nice embedding in which $C$ is a facial cycle.

\section{Tutte paths}

The main objective of this section is to prove several lemmas about Tutte subgraphs in planar graphs. The following two results will be used frequently. The first is due to Thomassen [6], and the second is due to Thomas and $\mathrm{Yu}$ [4].

(3.1) Lemma. Let $G$ be a 2-connected plane graph, let $C$ be a facial cycle of $G$, and let $u \in V(C)$, $e \in E(C)$, and $v \in V(G)-\{u\}$. Then $G$ contains a $C$-Tutte path $P$ from $u$ to $v$ and through $e$.

Note that Lemma (3.1) holds for connected graphs as long as $C$ is a facial walk and $G$ contains a path from $x$ to $y$ and through $e$. 
(3.2) Lemma. Let $G$ be a 2-connected plane graph, and let $C$ be a facial cycle of $G$. Let $u, v \in V(C)$ be distinct, let $e, f \in E(C)$, and assume that $u, v, e, f$ occur on $C$ in this clockwise order. Then $G$ contains a $v C u$-Tutte path $P$ from $u$ to $v$ and through $e$ and $f$.

Next, we prove a technical lemma, which will be used many times in later proofs. This lemma is stated in a fairly general setting in order to cover all situations in which it is applied. See Figure 1 for an illustration.

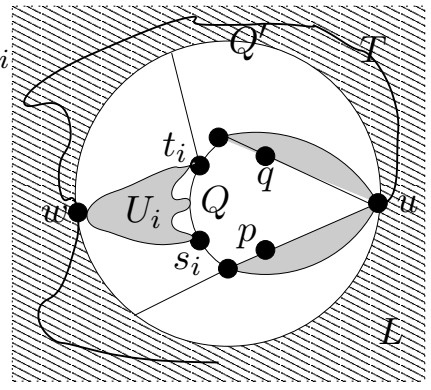

(a) $Q^{\prime}$ is a cycle

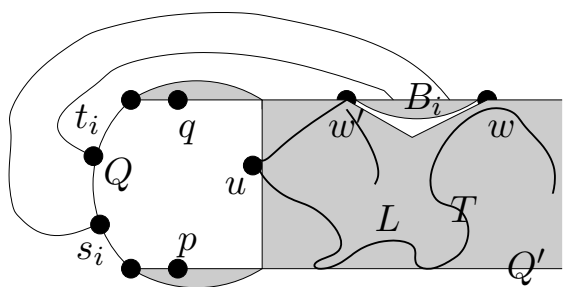

(b) $Q^{\prime}$ is a path or 2-way infinite path

Figure 1: Illustration of Lemma (3.3)

(3.3) Lemma. Let $K$ be a connected (finite or infinite) plane graph, $C$ be a facial walk of $K, Q$ be a path between $p$ and $q$ on $C, u \in V(C)-V(Q), L$ be a subgraph of $K-V(Q)$, and $Q^{\prime}$ be a cycle in $L$ or a path or a 2-way infinite path in $L$. Suppose the following three conditions are satisfied:

(1) for any $(L \cup Q)$-bridge $B$ of $K,|V(B \cap L)| \leq 1$ and $V(B \cap L) \subseteq V\left(Q^{\prime}\right)$,

(2) $K-V(L)$ is finite and all vertices of $K-V(L)$ has finite degree in $K$, and

(3) $L$ contains a $Q^{\prime}$-Tutte subgraph $T$ with $u \in V(T)$ and $\left|V\left(Q^{\prime}\right) \cap V(T)\right| \geq 2$.

Then $K-V(T)$ contains a path $S$ between $p$ and $q$ such that $S \cup T$ is a $Q$-Tutte subgraph of $K$, and every $T$-bridge of $L$ containing no edge of $Q^{\prime}$ is also an $(S \cup T)$-bridge of $K$.

Proof. Let $W$ denote the set of attachments on $Q^{\prime}$ of $(L \cup Q)$-bridges of $K$. By (2), $W$ is a finite set. Note that for each $w \in W$, either $w \in V(T)$ or there is a unique $T$-bridge $X$ of $L$ such that $w \in V(X)-V(T)$. For any $w, w^{\prime} \in W$, we define $w \sim w^{\prime}$ if $w=w^{\prime}$ or there is a $T$-bridge $X$ of $L$ such that $\left\{w, w^{\prime}\right\} \subseteq V(X)-V(T)$. Clearly, $\sim$ is an equivalence relation on $W$. Let $W_{1}, W_{2}, \ldots, W_{m}$ denote the equivalence classes of 
$W$ with respect to $\sim$. Then for each $i \in\{1, \ldots, m\}$, either $\left|W_{i}\right|=1$ and $W_{i} \subseteq V(T)$ (in this case, let $\left.B_{i}:=W_{i}\right)$ or $W_{i} \subseteq V\left(B_{i}\right)-V(T)$ for some $T$-bridge $B_{i}$ of $L$. Since $T$ is a $Q^{\prime}$-Tutte subgraph of $L$ and $W \subseteq V\left(Q^{\prime}\right),\left|V\left(B_{i} \cap T\right)\right| \leq 2$. Hence, it follows from (3) that $V\left(B_{i} \cap T\right) \subseteq V\left(Q^{\prime}\right)$.

Next we describe subgraphs $T_{i}$ and $U_{i}$ of $K$ between $L$ and $Q$, and the desired path $S$ will be contained in the union of these subgraphs. For each $i \in\{1, \ldots, m\}$, let $s_{i}, t_{i} \in V(Q)$ such that (i) $p, s_{i}, t_{i}, q$ occur on $Q$ in order, (ii) there are $w_{s}, w_{t} \in W_{i}$ such that $\left\{s_{i}, w_{s}\right\}$ is contained in a $(L \cup Q)$-bridge of $K$ and $\left\{t_{i}, w_{t}\right\}$ is contained in a $(L \cup Q)$ bridge of $K$, and (iii) subject to (i) and (ii), $s_{i} Q t_{i}$ is maximal. (See Figure 1(a) when $\left|W_{i}\right|=1$ and Figure $1(\mathrm{~b})$ when $\left|W_{i}\right| \geq 2$.) By planarity and since $u \in V(C)$ and $Q$ is a path on $C$, the paths $s_{i} Q t_{i}, i=1, \ldots, m$, are edge disjoint. We may therefore assume that $p, s_{1}, t_{1}, s_{2}, t_{2}, \ldots, s_{m}, t_{m}, q$ occur on $Q$ in this order. For each $i \in\{1, \ldots, m\}$, let $U_{i}$ denote the union of $s_{i} Q t_{i}, B_{i}$, and those $(L \cup Q)$-bridges of $K$ whose attachments are all contained in $V\left(s_{i} Q t_{i}\right) \cup W_{i}$. Let $t_{0}:=p$ and $s_{m+1}:=q$. For each $i \in\{0, \ldots, m\}$, let $T_{i}$ denote the union of $t_{i} Q s_{i+1}$ and those $(L \cup Q)$-bridges of $K$ whose attachments are all contained in $V\left(t_{i} Q s_{i+1}\right)$. Note that there is no path from $T_{i}-\left\{t_{i}, s_{i+1}\right\}$ to $L$ in $K-\left\{t_{i}, s_{i+1}\right\}$. By the definition of $s_{i} Q t_{i}$, the graphs $U_{i}$ and $T_{j}$ are almost disjoint. More precisely, we have the following.

(a) For any $i \leq j, U_{i} \cap T_{j}$ (and for $i<j,\left(U_{i}-T\right) \cap\left(U_{j}-T\right)$ ) is one of the following: $\emptyset$, or $\left\{t_{i}\right\}$, or the union of those $(L \cup Q)$-bridges of $K$ with $t_{i}$ as their only attachment on $L \cup Q$. Similarly, for $i<j, T_{i} \cap T_{j}$ (and also $T_{i} \cap U_{j}$ ) is one of the following: $\emptyset$, or $\left\{s_{i+1}\right\}$, or the union of those $(L \cup Q)$-bridges of $K$ with $s_{i+1}$ as their only attachment on $L \cup Q$.

Next we show how to route the desired path $S$ through $T_{i}$.

(b) For each $i \in\{0, \ldots, m\}, T_{i}$ contains a $t_{i} Q s_{i+1}$-Tutte path $R_{i}$ between $t_{i}$ and $s_{i+1}$.

If $\left|V\left(t_{i} Q s_{i+1}\right)\right| \leq 2$, then $R_{i}:=t_{i} Q s_{i+1}$ gives the desired path for (b). Now assume that $\left|V\left(t_{i} Q s_{i+1}\right)\right| \geq 3$. Let $C_{i}:=t_{i} Q s_{i+1}+t_{i} s_{i+1}$ and choose an edge $e$ from $E\left(t_{i} Q s_{i+1}\right)$. Note that $T_{i}+t_{i} s_{i+1}$ has a plane representation in which $C_{i}$ is a facial cycle. By applying Lemma (3.1) (with $T_{i}+t_{i} s_{i+1}, C_{i}, t_{i}, s_{i+1}$ as $G, C, u, v$, respectively), $T_{i}+t_{i} s_{i+1}$ has a $C_{i}$-Tutte path $R_{i}$ between $t_{i}$ and $s_{i+1}$ such that $e \in E\left(R_{i}\right)$. Clearly, $R_{i}$ is a $t_{i} Q s_{i+1}$-Tutte path in $T_{i}$.

Now we show how to route the desired path $S$ through $U_{i}$.

(c) For each $i \in\{1, \ldots, m\}, U_{i}-V\left(T \cap U_{i}\right)$ contains a path $S_{i}$ between $s_{i}$ and $t_{i}$ such that $S_{i} \cup\left(U_{i} \cap T\right)$ is an $s_{i} Q t_{i}$-Tutte subgraph of $U_{i}$.

Note that for all $i \in\{1, \ldots, m\},\left|V\left(U_{i} \cap T\right)\right|=\left|V\left(B_{i} \cap T\right)\right| \leq 2$. If $s_{i}=t_{i}$, then let $S_{i}:=s_{i} Q t_{i}$, and clearly, $S_{i} \cup\left(U_{i} \cap T\right)$ is an $s_{i} Q t_{i}$-Tutte subgraph of $U_{i}$ (because $\left.\left|V\left(U_{i} \cap T\right)\right| \leq 2\right)$. So assume that $s_{i} \neq t_{i}$. We distinguish two cases.

First assume that $W_{i} \subseteq V(T)$. Then $\left|W_{i}\right|=1$. Let $w$ be the only vertex in $W_{i}$. See Figure 1(a). Clearly, $U_{i}+t_{i} w$ has a plane representation so that $s_{i} Q t_{i}+\left\{w, t_{i} w\right\}$ is 
contained in a facial walk $D_{i}$ of $U_{i}+t_{i} w$. By Lemma (3.1) (with $U_{i}+t_{i} w, D_{i}, s_{i}, w, t_{i} w$ as $G, C, u, v, e$, respectively), $U_{i}+t_{i} w$ contains a $D_{i}$-Tutte path $S_{i}^{\prime}$ between $s_{i}$ and $w$ such that $t_{i} w \in E\left(S_{i}^{\prime}\right)$. Let $S_{i}:=S_{i}^{\prime}-w$. Then $S_{i} \subseteq U_{i}-V\left(T \cap U_{i}\right)$, and it is easy to see that $S_{i} \cup\left(U_{i} \cap T\right)=S_{i} \cup\{w\}$ is an $s_{i} Q t_{i}$-Tutte subgraph of $U_{i}$.

Now assume that $W_{i} \not \subseteq V(T)$. Then $W_{i} \subseteq V\left(B_{i}\right)-V(T)$ for some $T$-bridge $B_{i}$ of $L$ containing an edge of $Q^{\prime}$. Hence, since $Q^{\prime}$ is either a cycle or a 2-way infinite path, it follows from (3) that $V\left(B_{i} \cap T\right)$ consists of exactly two vertices, say $w$ and $w^{\prime}$. Assume that $w, w^{\prime}, t_{i}, s_{i}$ occur on the outer walk of $U_{i}$ in cyclic order. See Figure 1(b). Note that $s_{i} Q t_{i}+\left\{w, w^{\prime}, w s_{i}, t_{i} w^{\prime}\right\}$ is contained in a cycle of $U_{i}+\left\{w s_{i}, t_{i} w^{\prime}\right\}$, and hence, let $U_{i}^{\prime}$ denote a plane representation of the block of $U_{i}+\left\{w s_{i}, t_{i} w^{\prime}\right\}$ containing one such cycle. Without loss of generality, we may assume that $s_{i} Q t_{i}+\left\{w, w^{\prime}, w s_{i}, t_{i} w^{\prime}\right\}$ is contained in a facial cycle $D_{i}^{\prime}$ of $U_{i}^{\prime}$ such that $w, w^{\prime}, t_{i} w^{\prime}, w s_{i}$ occur on $D_{i}^{\prime}$ in clockwise order. By Lemma (3.2) (with $U_{i}^{\prime}, D_{i}^{\prime}, w, w^{\prime}, t_{i} w^{\prime}, w s_{i}$ as $G, C, u, v, e, f$, respectively), $U_{i}^{\prime}$ contains a $w^{\prime} D_{i}^{\prime} w$-Tutte path $S_{i}^{\prime}$ between $w$ and $w^{\prime}$ such that $\left\{w s_{i}, t_{i} w^{\prime}\right\} \subseteq E\left(S_{i}^{\prime}\right)$. Clearly, $S_{i}^{\prime}$ is also an $s_{i} Q t_{i}$-Tutte path in $U_{i}+\left\{w s_{i}, t_{i} w^{\prime}\right\}$. Let $S_{i}:=S_{i}^{\prime}-\left\{w, w^{\prime}\right\}$. Then $S_{i} \subseteq U_{i}-V\left(T \cap U_{i}\right)$, and it is easy to see that $S_{i} \cup\left(U_{i} \cap T\right)=S_{i} \cup\left\{w, w^{\prime}\right\}$ is an $s_{i} Q t_{i}$-Tutte subgraph of $U_{i}$.

By (a), (b) and (c), $S:=\left(\bigcup_{i=0}^{m} R_{i}\right) \cup\left(\bigcup_{i=1}^{m} S_{i}\right)$ is a path between $p$ and $q$ in $K-V(T)$. It is easy to see that every non-trivial $(S \cup T)$-bridge of $K$ is one of the following: a $T$ bridge of $L$ not contained in any $U_{i}$, or a $R_{i}$-bridge of $T_{i}$, or an $\left(S_{i} \cup\left(U_{i} \cap T\right)\right)$-bridge of $U_{i}$, or a $(L \cup Q)$-bridge of $K$ with only one attachment that is $s_{i}$ or $t_{i}$. Thus, $S \cup T$ is a $Q$-Tutte subgraph of $K$, and every $T$-bridge of $L$ containing no edge of $Q^{\prime}$ (and hence not contained in any $\left.U_{i}\right)$ is also an $(S \cup T)$-bridge of $K$.

Our next lemma deals with disjoint paths that form a Tutte subgraph in a planar graph. See Figure 2 for an illustration.

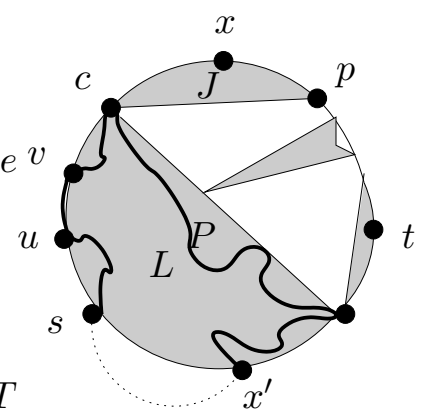

Figure 2: Illustration of Lemma (3.4)

(3.4) Lemma. Let $G$ be a 2-connected plane graph with a facial cycle $C$, let $s, u, v, x, t, x^{\prime}$ be vertices on $C$ in clockwise order. Suppose that $u v \in E(C), t \neq x^{\prime} \neq s$, 
$v \neq x$, and $G-V(x C t)$ contains a path from $s$ to $x^{\prime}$ and through $u v$. Then $G$ contains disjoint paths $P$ and $Q$ such that $P$ is from $s$ to $x^{\prime}$ and through $u v, Q$ is from $x$ to $t$, and $P \cup Q$ is an $s C t$-Tutte subgraph of $G$.

Proof. Without loss of generality, assume that $C$ is the outer cycle of $G$ (that is, $C=\partial G$ ). Let $L$ be the minimal subgraph of $G-V(x C t)$ such that $L$ is a union of blocks of $G-V(x C t)$ and $L$ contains a path from $s$ to $x^{\prime}$. Then all paths in $G-V(x C t)$ between $x^{\prime}$ and $s$ are contained in $L$, and hence, $x^{\prime} C s \subseteq L$ and $u v \in E(L)$. Let $c$ be the vertex of $C \cap L$ such that $c C x$ is minimal. Let $L^{\prime}:=L+x^{\prime} s$, where the edge $x^{\prime} s$ is added in such a way that $E(s C c) \cup\left\{x^{\prime} s\right\} \subseteq \partial L^{\prime}$ (shown in Figure 2 as a dotted edge). By the minimality of $L$, every cut vertex of $L$ (if any) must separate $s$ from $x^{\prime}$. Therefore, $L^{\prime}$ is 2-connected.

Observe that, since $L$ is a union of blocks of $G-V(x C t)$, each $(L \cup x C t)$-bridge of $G$ has at most one attachment on $c \partial L^{\prime} x^{\prime}$, with its remaining attachments on $x C t$. In $L^{\prime}$, we use Lemma (3.2) to find an $s \partial L^{\prime} x^{\prime}$-Tutte path $P$ from $x^{\prime}$ to $s$ and through $u v$ and $c$ (by choosing an edge of $C$ incident with $c$ ). Next, we will find the path $Q$, which is done in two steps.

Let $p \in V(x C t)$ with $x C p$ maximal such that $\{p, c\}$ is contained in a $(L \cup x C t)$-bridge of $G$. Let $J$ denote the union of $x C p$ and those $(L \cup x C t)$-bridges of $G$ whose attachments are all contained in $V(x C p) \cup\{c\}$. If $x=p$ then let $R$ denote the trivial path consisting of $x$; if $x \neq p$ then by Lemma (3.1) there is a $c C p$-Tutte path $R^{\prime}$ in $J+p c$ from $c$ to $x$ and through $p c$, and let $R:=R^{\prime}-c$.

It is easy to verify that the conditions of Lemma (3.3) hold, with $G^{\prime}:=G-V(J-$ $\{c, p\}), L, p C t, s \partial L^{\prime} x, c, P$ as $K, L, Q, Q^{\prime}, u, T$, respectively. Hence by Lemma (3.3), there is a path $S$ from $p$ to $t$ in $G^{\prime}-V(P)$ such that $S \cup P$ is a $p C t$-Tutte subgraph of $G^{\prime}$, and every $P$-bridge of $L$ containing no edge of $Q^{\prime}$ is also an $(S \cup P)$-bridge of $G^{\prime}$. In fact, it follows from planarity and $\left\{s, c, x^{\prime}\right\} \subseteq V(P)$ that any $P$-bridge of $L$ containing an edge of $s C c$ is also an $(S \cup P)$-bridge of $G^{\prime}$, and so, has at most two attachments on $S \cup P$.

Let $Q=R \cup S$. Clearly, $P \cap Q=\emptyset$ and $Q$ is a path from $x$ to $t$. It is easy to see that any non-trivial $(P \cup Q)$-bridge of $G$ is either an $(S \cup P)$-bridge of $G^{\prime}$ or a $R^{\prime}$-bridge of $J+p c$. Hence, $P \cup Q$ is an $s C t$-Tutte subgraph of $G$.

We conclude this section by proving three technical lemmas, which will be used to extend Tutte paths in a subgraph of a graph $G$ to Tutte paths in $G$. In order to cover all the situations, the lemmas are stated in a fairly general setting. Fortunately, their proofs are quite simple, with the help of Lemma (3.3). For an illustration of the next result, see Figure 3.

(3.5) Lemma. Let $G$ be a 2-connected (finite or infinite) plane graph and $C$ be a facial cycle of $G$ such that $G$ is $(4, C)$-connected. Let $x, u, v \in V(C)$ and $u v \in E(C)$ with 


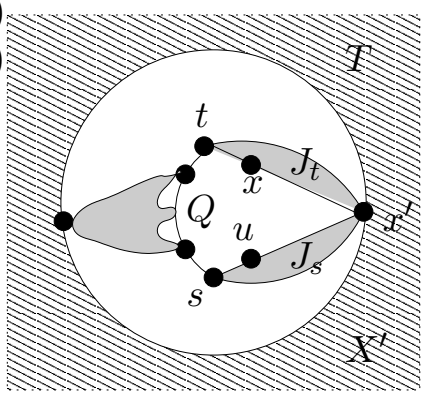

(a) $\left|V(C) \cap V\left(C^{\prime}\right)\right|=1$

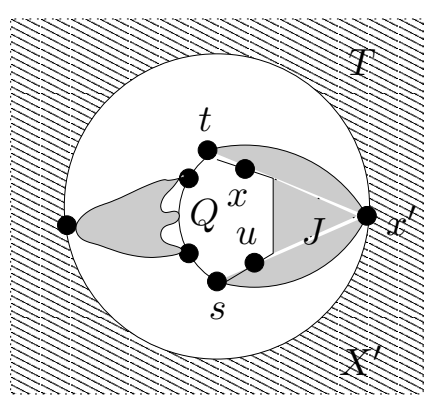

(b) $\left|V(C) \cap V\left(C^{\prime}\right)\right|=0$

Figure 3: Illustration of Lemma (3.5).

$v \neq x, Q$ be the subpath of $C-v$ between $x$ and $u$, and $G^{\prime}$ be a block of $G-V(Q)$ such that

(i) $v$ and $G^{\prime}$ are in the same component of $G-V(Q)$,

(ii) $G^{\prime}$ has a facial cycle $C^{\prime}$ for which $I_{G}\left(C^{\prime}\right)$ is defined and $C \subseteq I_{G}\left(C^{\prime}\right)$, and

(iii) $\left|V\left(C \cap C^{\prime}\right)\right| \leq 1$.

Then there exists $x^{\prime} \in V\left(C^{\prime}\right)$ such that, for any (finite or infinite) subgraph $X$ of $G$ containing $I_{G}\left(C^{\prime}\right)$ and for any (finite or 1-way infinite) $C^{\prime}$-Tutte path $P^{\prime}$ in $X^{\prime}:=X \cap G^{\prime}$ from $x^{\prime}$ with $\left|V\left(P^{\prime} \cap C^{\prime}\right)\right| \geq 2$, there is a $C$-Tutte path $P$ in $X$ from $x$ and through uv with the following properties:

(a) $P^{\prime} \subseteq P$,

(b) $u \in V(x P v)$ and $P-V\left(P^{\prime}-x^{\prime}\right)$ is a path from $x$ to $x^{\prime}$, and

(c) for any $z \in V(P)-V\left(P^{\prime}\right)$, either $z \notin V\left(X^{\prime}\right)$ or $z \in V(Z)-V\left(P^{\prime}\right)$ for some $P^{\prime}$-bridge $Z$ of $X^{\prime}$ containing an edge of $C^{\prime}$.

Proof. Without loss of generality, assume that $u C x=Q$ if $u \neq x$. (If $u=x$ then $Q$ is just the trivial path consisting of $u=x$ only.) Since $C^{\prime} \subseteq G-V(Q), C \cap C^{\prime} \subseteq C-V(Q)$. If $\left|V\left(C \cap C^{\prime}\right)\right|=1$, then let $x^{\prime}$ be the unique vertex of $C \cap C^{\prime}$. See Figure 3(a). Now assume that $\left|V\left(C \cap C^{\prime}\right)\right|=0$. Then from planarity, $C-V(Q)$ is contained in a single $\left(G^{\prime} \cup Q\right)$-bridge of $G$. Let $x^{\prime}$ be the attachment on $C^{\prime}$ of this $\left(G^{\prime} \cup Q\right)$-bridge of $G ; x^{\prime}$ exists because $v$ and $G^{\prime}$ are contained in the same component of $G-V(Q)$. See Figure 3(b). 
Since $G$ is $(4, C)$-connected, there exist distinct $s, t \in V(Q)$ such that $u, s, t, x$ occur on $Q$ in order, $\left\{s, x^{\prime}\right\}$ is contained in a $\left(G^{\prime} \cup Q\right)$-bridge of $G,\left\{t, x^{\prime}\right\}$ is contained in a $\left(G^{\prime} \cup Q\right)$-bridge of $G$, and no $\left(G^{\prime} \cup Q\right)$ of $G$ containing $x^{\prime}$ contains any vertex of $s Q t-\{s, t\}$. Let $J$ denote the union of $t C s$ and those $\left(G^{\prime} \cup Q\right)$-bridges of $G$ whose attachments are all contained in $V(t C s) \cup\left\{x^{\prime}\right\}$.

Next we show that $J$ contains disjoint paths $P_{s}$ and $P_{t}$ such that $P_{s}$ is from $x^{\prime}$ to $s$ and through $u v, P_{t}$ is from $x$ to $t, u \in V\left(s P_{s} v\right), P_{s} \cup P_{t}$ is a $t C s$-Tutte subgraph of $J$.

First, assume that $\left|V\left(C \cap C^{\prime}\right)\right|=1$. See Figure 3(a). Then $x^{\prime}$ is a cut vertex of $J$. Let $J_{s}, J_{t}$ denote the subgraphs of $J$ such that $J_{s} \cup J_{t}=J, V\left(J_{s} \cap J_{t}\right)=\left\{x^{\prime}\right\}, x^{\prime} C s \subseteq J_{s}$ and $t C x^{\prime} \subseteq J_{t}$. In $J_{s}+x^{\prime} s$, we apply Lemma (3.1) to find an $x^{\prime} C s$-Tutte path $P_{s}$ from $x^{\prime}$ to $s$ and through $u v$. By planarity, $u \in V\left(s P_{s} v\right)$. If $t=x$ then let $P_{t}$ denote the trivial path consisting of $x$; and if $t \neq x$ then we use Lemma (3.1) to find a $t C x^{\prime}$-Tutte path $P_{t}^{\prime}$ in $J_{t}+x^{\prime} t$ from $x$ to $x^{\prime}$ and through $t x^{\prime}$, and let $P_{t}:=P_{t}^{\prime}-x^{\prime}$. It is easy to verify that $P_{s}$ and $P_{t}$ give the desired paths.

Now assume $\mid V\left(C \cap C^{\prime}\right)=0$. See Figure 3(b). Since $G$ is 2-connected and $s \neq t$, $J^{\prime}:=J+\left\{x^{\prime} s, x^{\prime} t\right\}$ is 2 -connected. Clearly, $J^{\prime}$ has a plane representation so that $\partial J^{\prime}=$ $t C s+\left\{x^{\prime}, x^{\prime} s, x^{\prime} t\right\}$, and $x^{\prime}, s, u, v, x, t$ occur on $\partial J^{\prime}$ in clockwise order. Thus $s \partial J^{\prime} t=t C s$. Since $v$ and $G^{\prime}$ are contained in a component of $G-V(Q), J^{\prime}-V(Q)$ has a path from $v$ to $x^{\prime}$. Hence, $J^{\prime}-V(t C x)$ contains a path from $s$ to $x^{\prime}$ and through $u v$. By Lemma (3.4) (with $J^{\prime}, \partial J^{\prime}$ as $G, C$, respectively), $J^{\prime}$ contains disjoint paths $P_{s}$ and $P_{t}$ such that $P_{s}$ is from $x^{\prime}$ to $s$ and through $u v, P_{t}$ is from $x$ to $t$, and $P_{s} \cup P_{t}$ is an $s \partial J^{\prime} t$-Tutte subgraph of $J^{\prime}$. By planarity, $u \in V\left(s P_{s} v\right)$. It is easy to see that $P_{s}$ and $P_{t}$ give the desired paths.

To complete the proof this lemma, let $X$ be a subgraph of $G$ containing $I_{G}\left(C^{\prime}\right)$ and let $P^{\prime}$ be a (finite or 1-way infinite) $C^{\prime}$-Tutte path in $X^{\prime}:=X \cap G^{\prime}$ from $x^{\prime}$ with $V\left(P^{\prime} \cap C^{\prime}\right) \mid \geq 2$. Note that $x^{\prime}$ is on the facial walk of $X-V\left(J-\left\{s, t, x^{\prime}\right\}\right)$ containing $Q$. It is straightforward to verify that the conditions of Lemma (3.3) hold, with $X^{*}:=X-$ $V\left(J-\left\{s, t, x^{\prime}\right\}\right), X^{\prime}, P^{\prime}, s Q t, s, t, C^{\prime}, x^{\prime}$ as $K, L, T, Q, p, q, Q^{\prime}, u$, respectively. By Lemma (3.3), we find a path $S$ from $s$ to $t$ in $X^{*}-V\left(P^{\prime}\right)$ such that $S \cup P^{\prime}$ is a $s Q t$-Tutte subgraph of $X^{*}$ and every $P^{\prime}$-bridge of $X^{\prime}$ containing no edge of $C^{\prime}$ is an $\left(S \cup P^{\prime}\right)$-bridge of $X^{*}$.

Let $P=P^{\prime} \cup S \cup P_{s} \cup P_{t}$. Then $P$ is a path from $x, P^{\prime} \subseteq P, u \in V(x P v)$, and $P-V\left(P^{\prime}-x^{\prime}\right)=S \cup P_{s} \cup P_{t}$ is a path between $x$ and $x^{\prime}$. Note that each non-trivial $P$-bridge of $X$ is one of the following: a $P^{\prime}$-bridge of $X^{\prime}$ not containing any edge of $C^{\prime}$, or an $\left(S \cup P^{\prime}\right)$-bridge of $X^{*}$, or a $\left(P_{s} \cup P_{t}\right)$-bridge of $J$. Hence, it is easy to check that $P$ is a $C$-Tutte path from $x$ and through $u v$ in $X$, which satisfies (a), (b), and (c).

For the next result, see Figure 4 for an illustration.

(3.6) Lemma. Let $G$ be a 2-connected (finite or infinite) plane graph and $C$ be a facial cycle of $G$ such that $G$ is $(4, C)$-connected. Let $x, u, v \in V(C)$ and $u v \in E(C)$ with $v \neq x, Q$ be the subpath of $C-v$ between $x$ and $u$, and $G^{\prime}$ be a block of $G-V(Q)$ such that 


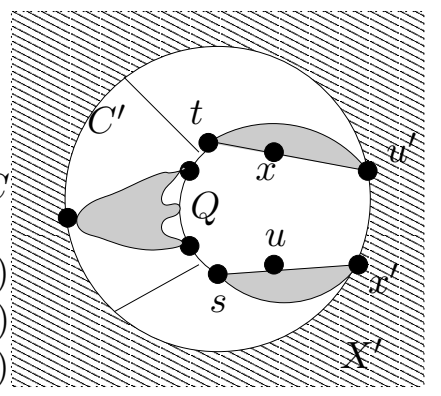

Figure 4: Illustration for Lemma (3.6).

(i) $v$ and $G^{\prime}$ are in the same component of $G-V(Q)$,

(ii) $G^{\prime}$ has a facial cycle $C^{\prime}$ for which $I_{G}\left(C^{\prime}\right)$ is defined and $C \subseteq I_{G}\left(C^{\prime}\right)$, and

(iii) $C \cap C^{\prime}$ is a non-trivial path.

Then there exist $x^{\prime} \in V\left(C \cap C^{\prime}\right)$ and $u^{\prime} v^{\prime} \in E\left(C^{\prime}\right)$ such that $x^{\prime}$ and $u^{\prime}$ are the endvertices of $C \cap C^{\prime}$, if $G$ is infinite then $v^{\prime}$ is in an infinite component of the graph obtained from $G$ by deleting the path in $C-v^{\prime}$ between $x^{\prime}$ and $u^{\prime}$, and, for any (finite or infinite) subgraph $X$ of $G$ containing $I_{G}\left(C^{\prime}\right)$ and for any (finite or 1-way infinite) $C^{\prime}$-Tutte path $P^{\prime}$ in $X^{\prime}:=X \cap G^{\prime}$ from $x^{\prime}$ and through $u^{\prime} v^{\prime}$, there is a $C$-Tutte path $P$ in $X$ from $x$ and through $u v$ with the following properties:

(a) $P^{\prime} \subseteq P$,

(b) $u \in V(x P v)$ and $P-V\left(P^{\prime}-x^{\prime}\right)$ is a path from $x$ to $x^{\prime}$, and

(c) for any $z \in V(P)-V\left(P^{\prime}\right)$, either $z \notin V\left(X^{\prime}\right)$ or $z \in V(Z)-V\left(P^{\prime}\right)$ for some $P^{\prime}$-bridge $Z$ of $X^{\prime}$ containing an edge of $C^{\prime}$.

Proof. Without loss of generality, assume that $Q=u C x$ if $u \neq x$. (If $u=x$ then $Q$ is the trivial path consisting of $u=x$ only.) Let $x^{\prime}$ and $u^{\prime}$ denote the endvertices of $C \cap C^{\prime}$ such that $x, u^{\prime}, x^{\prime}, v, u$ occur on $C$ in clockwise order. See Figure 4. Let $u^{\prime} v^{\prime} \in E\left(C^{\prime}\right)$ such that if $Q^{\prime}$ denotes the subpath of $C^{\prime}-v^{\prime}$ between $x^{\prime}$ and $u^{\prime}$ and if $G$ is infinite then the infinite component of $G^{\prime}-V\left(Q^{\prime}\right)$ contains $v^{\prime}$. Such $u^{\prime} v^{\prime}$ exists because $G$ is $(4, C)$-connected. (Note that there are only two choices for $v^{\prime}$, and if both do not work then $G-\left\{x, u^{\prime}\right\}$ has a component which does not contain any vertex of $C$.)

Let $s \in V(Q)$ with $u Q s$ maximal such that $\left\{s, x^{\prime}\right\}$ is contained in some $\left(G^{\prime} \cup Q\right)$ bridge of $G$, and let $J_{s}$ denote the union of $u Q s$ and those $\left(G^{\prime} \cup Q\right)$-bridges of $G$ whose 


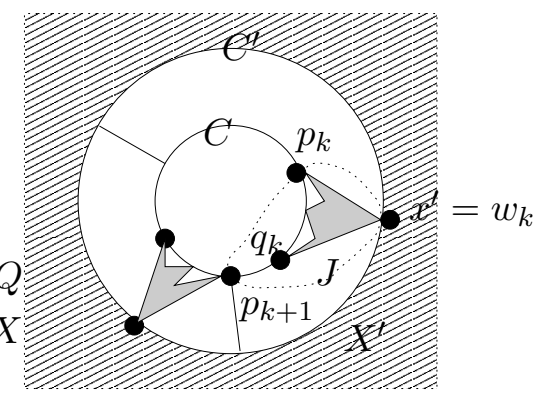

Figure 5: Illustration of Lemma (2.7).

attachments are all contained in $V(u Q s) \cup\left\{x^{\prime}\right\}$. By applying Lemma (3.1), we find an $x^{\prime} C s$-Tutte path $P_{s}$ in $J_{s}+x^{\prime} s$ from $x^{\prime}$ to $s$ and through $u v$. By planarity, $u \in V(x P v)$.

Let $t \in V(Q)$ with $t Q x$ maximal such that $\left\{t, u^{\prime}\right\}$ is contained in some $\left(G^{\prime} \cup Q\right)$ bridge of $G$, and let $J_{t}$ denote the union of $t Q x$ and those $\left(G^{\prime} \cup Q\right)$-bridges of $G$ whose attachments are all contained in $V(t Q x) \cup\left\{u^{\prime}\right\}$. If $t=x$ let $P_{t}$ be the path consisting of $x$ only; and if $t \neq x$ then by applying Lemma (3.1) we find a $t C x^{\prime}$-Tutte path $P_{t}^{\prime}$ in $J_{t}+t u^{\prime}$ from $u^{\prime}$ to $x$ through $t u^{\prime}$, and let $P_{t}:=P_{t}^{\prime}-u^{\prime}$.

To complete the proof, let $X$ be a subgraph of $G$ containing $I_{G}\left(C^{\prime}\right)$ and let $P^{\prime}$ be a (finite or 1-way infinite) $C^{\prime}$-Tutte path in $X^{\prime}:=X \cap G^{\prime}$ from $x^{\prime}$ and through $u^{\prime} v^{\prime}$. It is easy to verify that $X^{*}:=X-V\left(\left(J_{s} \cup J_{t}\right)-\left\{s, t, u^{\prime}, x^{\prime}\right\}\right), X^{\prime}, P^{\prime}, Q, s, t, C^{\prime}, x^{\prime}$ (as $K, L, T, Q, p, q, Q^{\prime}, u$ respectively) satisfy the conditions of Lemma (3.3). Hence by Lemma (3.3) we find a path $S$ from $s$ to $t$ in $X^{*}-V\left(P^{\prime}\right)$ such that $S \cup P^{\prime}$ is a $Q$-Tutte subgraph of $X^{*}$ and every $P^{\prime}$-bridge of $X^{\prime}$ containing no edge of $C^{\prime}$ is an $\left(S \cup P^{\prime}\right)$-bridge of $X^{*}$.

Let $P:=P^{\prime} \cup S \cup P_{s} \cup P_{t}$. Then $P^{\prime} \subseteq P, u \in V(x P v)$, and $P-V\left(P^{\prime}-x^{\prime}\right)=S \cup P_{s} \cup P_{t}$ is a path between $x$ and $x^{\prime}$. Note that each non-trivial $P$-bridge of $X$ is one of the following: a $P^{\prime}$-bridge of $G^{\prime}$ not containing any edge of $C^{\prime}$, or an $\left(S \cup P^{\prime}\right)$-bridge of $X^{*}$, or a $P_{s^{-}}$ bridge of $J_{s}+x^{\prime} s$, or a $P_{t}^{\prime}$-bridge of $J_{t}+t u^{\prime}$. Hence, it is easy to see that $P$ is a $C$-Tutte path from $x$ and through $u v$ in $X$ which satisfies (a), (b), and (c).

For an illustration of the final result in this section, see Figure 5.

(3.7) Lemma. Let $G$ be a 2-connected (finite or infinite) plane graph, $C$ be a facial cycle of $G$, and $x \in V(C)$ such that $G$ is $(4, C)$-connected. Let $G^{\prime}$ be a block of $G-V(C)$ and $C^{\prime}$ be a facial cycle of $G^{\prime}$ for which $I_{G}\left(C^{\prime}\right)$ is defined and $C \subseteq I_{G}\left(C^{\prime}\right)$. Then there exists $x^{\prime} \in V\left(C^{\prime}\right)$ such that, for any (finite or infinite) subgraph $X$ of $G$ containing $I_{G}\left(C^{\prime}\right)$ and for any (finite or 1-way infinite) $C^{\prime}$-Tutte path $P^{\prime}$ from $x^{\prime}$ in $X^{\prime}:=X \cap G^{\prime}$ with $\left|V\left(P^{\prime} \cap C^{\prime}\right)\right| \geq 2$, there is a $C$-Tutte path $P$ from $x$ in $X$ with the following properties: 
(a) $P^{\prime} \subseteq P$,

(b) $P-V\left(P^{\prime}-x^{\prime}\right)$ is a path between $x$ and $x^{\prime}$, and

(c) for any $z \in V(P)-V\left(P^{\prime}\right)$, either $z \notin V\left(X^{\prime}\right)$ or $z \in V(Z)-V\left(P^{\prime}\right)$ for some $P^{\prime}$-bridge $Z$ of $X^{\prime}$ containing an edge of $C^{\prime}$.

Proof. Let $w_{1}, \cdots, w_{m}$ be the attachments on $C^{\prime}$ of $\left(G^{\prime} \cup C\right)$-bridges of $G$ which occur on $C^{\prime}$ in clockwise order. Since $G$ is $(4, C)$-connected, there exist distinct $p_{j}, q_{j} \in V(C)$ such that (i) $\left\{p_{j}, w_{j}\right\}$ is contained in a $\left(G^{\prime} \cup C\right)$-bridge of $G$ and $\left\{q_{j}, w_{j}\right\}$ is contained in a $\left(G^{\prime} \cup C\right)$-bridge of $G$, (ii) every $\left(G^{\prime} \cup C\right)$-bridge of $G$ containing some $w_{l} \neq w_{j}$ contains no vertex of $p_{j} C q_{j}-\left\{p_{j}, q_{j}\right\}$, and (iii) subject to (i) and (ii), $p_{j} C q_{j}$ is maximal.

Without loss of generality, assume that $x \in V\left(p_{k} C p_{k+1}\right)-\left\{p_{k}\right\}$ for some $1 \leq k \leq m$, where $p_{m+1}:=p_{1}$. Let $J$ denote the union of $p_{k} C p_{k+1}$ and those $\left(G^{\prime} \cup C\right)$-bridges of $G$ whose attachments are all contained in $V\left(p_{k} C p_{k+1}\right) \cup\left\{w_{k}\right\}$. Let $x^{\prime}=w_{k}$. See Figure 5 .

Next we show that $J$ contains disjoint paths $P^{*}$ and $Q^{*}$ such that $P^{*}$ is from $x^{\prime}$ to $p_{k}, Q^{*}$ is from $x$ to $p_{k+1}$, and $P^{*} \cup Q^{*}$ is a $p_{k} C p_{k+1}$-Tutte subgraph of $J$. Since $G$ is 2 -connected, $J^{\prime}:=J+x^{\prime} p_{k+1}$ is 2 -connected. Clearly $J^{\prime}$ has a plane representation in which $p_{k} C p_{k+1}+\left\{x^{\prime}, x^{\prime} p_{k+1}\right\} \subseteq \partial J^{\prime}$, and $p_{k}, x^{\prime}, p_{k+1}, x$ occur on $\partial J^{\prime}$ in clockwise order. We use Lemma (3.1) to find a $\partial J^{\prime}$-Tutte path $P^{\prime \prime}$ in $J^{\prime}$ from $x$ to $p_{k}$ and through $x^{\prime} p_{k+1}$. Let $P^{*}$ and $Q^{*}$ be the components of $P^{\prime \prime}-x^{\prime} p_{k+1}$, where $P^{*}$ is a path from $x^{\prime}$ to $p_{k}$ and $Q^{*}$ is a path from $x$ to $p_{k+1}$. Clearly, every non-trivial $\left(P^{*} \cup Q^{*}\right)$-bridge of $J$ is a $P^{\prime \prime}$-bridge of $J$. Hence $P^{*} \cup Q^{*}$ is a $p_{k} C p_{k+1}$-Tutte subgraph of $J$.

Now we see that $X^{*}:=X-V\left(J-\left\{x^{\prime}, p_{k}, p_{k+1}\right\}\right), X^{\prime}, p_{k+1} C p_{k}, C^{\prime}, P^{\prime}, p_{k+1}, p_{k}, x^{\prime}$ (as $K, L, Q, Q^{\prime}, T, p, q, u$, respectively) satisfy the conditions of Lemma (3.3). By (3.3), we find a path $S$ from $p_{k+1}$ to $p_{k}$ in $X^{*}-V\left(P^{\prime}\right)$ such that $S \cup P^{\prime}$ is a $p_{k+1} C p_{k}$-Tutte subgraph of $X^{*}$ and any $P^{\prime}$-bridge of $X^{\prime}$ containing no edge of $C^{\prime}$ is also an $\left(S \cup P^{\prime}\right)$-bridge of $X^{*}$.

Let $P=P^{\prime} \cup S \cup\left(P^{*} \cup Q^{*}\right)$. Then each non-trivial $P$-bridge of $X$ is one of the following: a $P^{\prime}$-bridge of $G^{\prime}$ containing no edge of $C^{\prime}$, or a $\left(P^{*} \cup Q^{*}\right)$-bridge of $J$, or an $\left(S \cup P^{\prime}\right)$-bridge of $X^{*}$. Hence, it is easy to check that $P$ is a $C$-Tutte path in $G$.

Clearly, $P^{\prime} \subseteq P, P-V\left(P^{\prime}-x^{\prime}\right)=S \cup\left(P^{*} \cup Q^{*}\right)$ is a path between $x$ and $x^{\prime}$, and for any $z \in V(P)-V\left(P^{\prime}\right)$, either $z \notin V\left(X^{\prime}\right)$ or $z \in V(Z)-V\left(P^{\prime}\right)$ for some $P^{\prime}$-bridge $Z$ of $X^{\prime}$ containing an edge of $C^{\prime}$. Thus $P$ gives the desired path in $X$.

\section{Tutte paths in graphs with ladder nets}

We begin this section by stating Theorem (3.7) of [10], which will be used in the next section to deal with graphs with ladder nets. 
(4.1) Lemma. Let $G$ be a 2-connected, 2-indivisible, infinite, plane graph with a ladder net $N$. Let $x \in V(\partial N)$ and $u v \in E(\partial N)$ such that $u \in V(x \partial N v)$. Then $G$ contains a 1-way infinite $\partial N$-Tutte path $P$ from $x$ and through $u v$ such that $u \in V(x P v)$.

We devote the rest of this section to proving a lemma, which will be used as a part of the induction basis in the proof of Theorem (1.2). Before we do this, let us extend the notation $\partial G$ to infinite graphs. Let $G$ be an infinite plane graph. Then $\partial G$ denotes the subgraph of $G$ defined as follows: for every $x \in V(G) \cup E(G)$, we have $x \in \partial G$ if, and only if, for any cycle $C$ in $G$ for which $I(C)$ is defined, $x \notin I(C)-V(C)$.

Let $G$ be a 2 -connected, 2 -indivisible, infinite, plane graph, and let $C$ be a facial cycle of $G$ such that $G$ is $(4, C)$-connected. If $G$ has a radial net, then clearly $\partial G=\emptyset$. Now assume that $G$ does not have a radial net. Let $S$ be the set of vertices of infinite degree in $G$. Then $|S| \leq 2$, and there exists $F \subseteq E(G)$ as in Theorem (2.1) such that $G-F$ has a ladder net $N$ satisfying the conclusions of Theorem (2.1). If $S=\emptyset$, then $\partial G=\partial N$. If $|S|=2$, then $\partial G$ is the subpath of $\partial N$ between the vertices of $S$. If $|S|=1$ and one $S$-bridge of $\partial N$ contains all incident vertices of edges in $F$, then $\partial G$ is the other $S$-bridge of $\partial N$, which is a 1-way infinite path. If $|S|=1$ and each $S$-bridge of $\partial N$ contains infinitely many vertices incident with edges in $F$, then $\partial G=S$ consists of only one vertex. Hence, if $\partial G \neq \emptyset$, then $\partial G$ is a trivial path, or a 1-way infinite path, or a 2-way infinite path, and the endvertices of $\partial G$ are in $S$.

Next, we prove the main result of this section. See Figure 6 for an illustration.

(4.2) Lemma. Let $G$ be a 2-connected, 2-indivisible, infinite plane graph, and $C$ be a facial cycle of $G$ such that $G$ is $(4, C)$-connected. Let $x \in V(C)$ and $u v \in E(C)$ with $v \neq x$, and let $Q$ be the subpath of $C-v$ between $u$ and $x$ such that $Q \cap \partial G \neq \emptyset$ and $v$ is in the infinite component of $G-V(Q)$. Then $G$ contains a 1-way infinite $C$-Tutte path $P$ from $x$ such that $u v \in E(P)$ and $u \in V(x P v)$.

Proof. Without loss of generality, we may assume that $Q=u C x$ if $u \neq x$. (If $u=x$ then $Q$ is a trivial path.) Let $a, b \in V(Q \cap \partial G)$ such that $a \partial G b$ is maximal and $u, a, b, x$ occur on $C$ in clockwise order. Let $S$ denote the set of vertices of infinite degree in $G$. By Theorem (2.1), $|S| \leq 2$, and there is a set $F$ of edges such that

(1) for any $f \in F, f$ is incident with exactly one vertex in $S$,

(2) $G-F$ has a net $N=\left(C_{1}, C_{2}, \cdots\right), C \subseteq I\left(C_{1}\right), S \subseteq \partial N$, and, for any $f \in F$, both incident vertices of $f$ are contained in a common infinite $S$-bridge of $\partial N$,

(3) if $|S|=1$, then either one $S$-bridge of $\partial N$ contains all vertices incident with edges in $F$ or each $S$-bridge of $\partial N$ contains infinitely many vertices incident with edges in $F$, and 


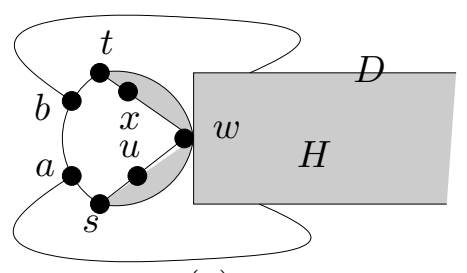

(a)

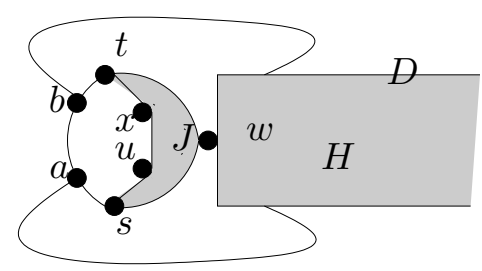

(b)

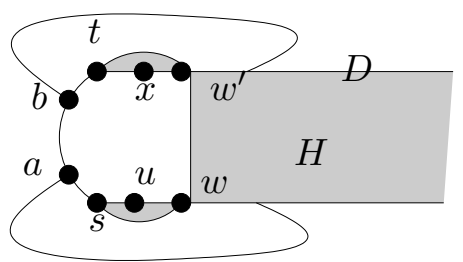

(c)

Figure 6: The structure of $G-F$.

(4) if $|S|=2$, then, for any $T \subseteq V(G)-S$ with $|T| \leq 3, S$ is contained in a component of $(G-F)-T$.

Since $Q \cap \partial G \neq \emptyset, N$ is a ladder net. Therefore, $(G-F)-V(Q)$ has a unique infinite block, say $H$, and $H$ has a ladder net, say $N_{H}$. See Figure 6 . For convenience, let $D:=\partial N_{H}$. Note that $D \cap \partial N$ has exactly two components. Moreover, the components of $D \cap \partial N$ are 1-way infinite paths, and between them there are infinitely many vertex disjoint paths (contained in $C_{i+1}-V\left(C_{i} \cap C_{i+1}\right)$ for all large $i$ ).

We claim that we may further choose $F$ such that

(5) $S \subseteq\{a, b\} \cup V(D \cap \partial N)$, every edge in $F$ has an incident vertex on $D \cap \partial N$, if $|S \cap V(D)|=1$ then there are at least three paths in $H$ from $S \cap V(D)$ to the component of $D \cap \partial N$ not containing $S \cap V(D)$ which only share the vertex in $S \cap V(D)$, and if $|S \cap V(D)|=2$ then there are at least three internally disjoint paths in $H$ between the vertices in $S \cap V(D)$.

This can be shown as follows. Since $S \subseteq V(\partial G)$ and since $a \partial G b$ is maximal subject to $a, b \in V(Q \cap \partial G)$, we see that $S \cap V(a \partial N b) \subseteq\{a, b\}$. If $S \subseteq\{a, b\}$, then let $F^{\prime}$ be obtained from $F$ by deleting all edges with no incident vertex on $D$ (there are only finitely many such edges), and we have (1)-(5) with $F^{\prime}$ replacing $F$. So assume $S \nsubseteq\{a, b\}$. For each $s \in S-V(a \partial N b)$, we choose $s^{\prime} \in V(D \cap \partial N)$ such that $s s^{\prime} \in F$ and there are sufficiently many disjoint paths in $G-F$ from $D \cap s \partial N s^{\prime}$ to the component of $D \cap \partial N$ not intersecting $s \partial N s^{\prime}$. (This can be done since $N$ and $N_{H}$ are ladder nets.) Let $F^{\prime}$ be 
obtained from $F$ by deleting all edges in $F$ whose incident vertices are all contained in $s \partial N s^{\prime}$, for all $s \in S-V(a \partial N b)$. Then $S-V(a \partial N b)$ is contained in a unique infinite block $H^{\prime}$ of $G-F^{\prime}$, and $H^{\prime}$ has a ladder net, say $N^{\prime}$. Let $D^{\prime}:=\partial N^{\prime}$. So $H \subseteq H^{\prime}$ and $S-V(a \partial N b) \subseteq V\left(D^{\prime}\right)$. Clearly, all edges in $F^{\prime}$ has an incident vertex in $D^{\prime}$. It is straightforward to verify that (1)-(5) are satisfied, with $F^{\prime}, N^{\prime}, D^{\prime}$ replacing $F, N, D$, respectively.

By planarity, the attachments on $H$ of $(H \cup Q)$-bridges of $G-F$ are all contained in $D$. Note that $D \cap C=\emptyset$ or $D \cap C$ is a path, and hence we distinguish two cases.

Case 1. $|V(D \cap C)| \leq 1$.

Then $D \cap C=\emptyset$ or $D \cap C$ is a trivial path. If $D \cap C \neq \emptyset$, then let $w$ denote the unique vertex of $D \cap C$. See Figure 6(a).

If $D \cap C=\emptyset$, then $C-V(Q-\{x, u\})$ is contained in a single $(H \cup Q)$-bridge $B_{v}$ of $G-F$. See Figure 6(b). In this case, we claim that $\left(B_{v}-V(Q)\right) \cap H \neq \emptyset$. For otherwise, $B_{v}-V(Q)$ is a finite component of $(G-F)-V(Q)$. Since $S \subseteq\{a, b\} \cup V(\partial N \cap D)$ (by (5)), $B_{v}-V(Q)$ is also a finite component of $G-V(Q)$. Therefore, $v$ is not in the infinite component of $G-V(Q)$, a contradiction. Hence, let $w \in V\left(B_{v}\right) \cap V(H)$. Then $w$ is the attachment of $B_{v}$ on $H$, and $B_{v}-V(Q)$ contains a path from $v$ to $w$.

In $H$, we use Lemma (4.1) to find a 1-way infinite $D$-Tutte path $P^{\prime}$ from $w$ such that if $(S-\{w\}) \cap V(D) \neq \emptyset$ then $P^{\prime}$ contains a vertex in $S-\{w\}$ (by using an edge of $D$ incident with that vertex $)$.

We claim that $S \cap V(D) \subseteq V\left(P^{\prime}\right)$. This is obvious when $w \in S$ (because $|S| \leq 2$ ) or when $|S \cap V(D)| \leq 1$. So assume that $w \notin S$ and $S \cap V(D)=\left\{s_{1}, s_{2}\right\}$. By planarity and since $Q \cap \partial G \neq \emptyset, s_{1}$ and $s_{2}$ belong to different components of $D \cap \partial N$. Suppose $s_{1} \notin V\left(P^{\prime}\right)$. Then $s_{1} \in V(B)$ for some $P^{\prime}$-bridge $B$ of $H$. Since $P^{\prime}$ is a $D$-Tutte path, $\left|V\left(B \cap P^{\prime}\right)\right|=2$. Note that $s_{2} \in V\left(P^{\prime}\right)$, because $P^{\prime}$ contains a vertex of $(S \cap V(D))-\{w\}$. Thus, $s_{2} \notin V(B)$ because $s_{2} \neq w$ and $s_{1}$ and $s_{2}$ belong to different components of $D \cap \partial N$. Therefore, $s_{1}$ and $s_{2}$ belong to different components of $H-V\left(B \cap P^{\prime}\right)$, contradicting (5).

We wish to extend $P^{\prime}$ to the desired path $P$. Let $s, t \in V(Q)$ with $a, b, t, x, u, s$ on $C$ in clockwise order such that (i) $\{s, w\}$ is contained in an $(H \cup Q)$-bridge of $G-F$ and $\{t, w\}$ is contained in an $(H \cup Q)$-bridge of $G-F$, (ii) every $(H \cup Q)$-bridge of $G-F$ containing a vertex of $W-\{w\}$ contains no vertex of $t C s-\{s, t\}$, and (iii) subject to (i) and (ii), $t C s$ is maximal. See Figure 6(a) and Figure 6(b). Then $t \neq s$; otherwise, $\{t=s, w\}$ is a 2-cut of $G$ and $G-\{s, w\}$ has a component containing no vertex of $C$, contradicting the assumption that $G$ is $(4, C)$-connected. Let $J$ denote the union of $t C s$ and those $(H \cup Q)$-bridges of $G-F$ whose attachments are all contained in $V(t C s) \cup\{w\}$.

Next we show that $J$ contains disjoint paths $P_{s}$ and $P_{t}$ such that $P_{s}$ is from $w$ to $s$ and through $u v, P_{t}$ is from $x$ to $t, u \in V\left(s P_{s} v\right)$, and $P_{s} \cup P_{t}$ is a $t C s$-Tutte subgraph of $J$. We consider two cases. 
First, assume $w \notin C$. Then $J^{\prime}:=J+\{w s, w t\}$ is 2-connected. Clearly, $J^{\prime}$ has a plane representation so that $\partial J^{\prime}=t C s+\{w, w s, w t\}$. Also $J^{\prime}-V(Q)$ has a path from $v$ to $w$ (because $B_{v}-V(Q)$ contains a path from $v$ to $\left.w\right)$. Hence, $J^{\prime}-V(t C x)$ contains a path from $s$ to $w$ and through $u v$. By applying Lemma (3.4) to $J^{\prime}$ (with $J^{\prime}, \partial J^{\prime}, w$ as $G, C, x^{\prime}$, respectively), we find disjoint paths $P_{s}$ and $P_{t}$ in $J^{\prime}$ such that $P_{s}$ is from $w$ to $s$ and through $u v, P_{t}$ is from $x$ to $t, P_{s} \cup P_{t}$ is a $s \partial J^{\prime} t$-Tutte subgraph of $J^{\prime}$. Note that $s \partial J^{\prime} t=t C s$, and $w s, w t \notin P_{s} \cup P_{t}$. Hence $P_{s} \cup P_{t}$ is a $t C s$-Tutte subgraph of $J$. By planarity, $u \in V\left(s P_{s} v\right)$.

Now assume $w \in C$. Then $w$ is a cut vertex of $J$. If $|V(w C s)|=2$ then let $P_{s}:=w C s$, and if $x=t$ then let $P_{t}$ denote the trivial path consisting of $x$ only. Now assume that $|V(w C s)| \geq 3$ and $x \neq t$. Since $G$ is $(4, C)$-connected, $J$ has exactly two $w$-bridges $J_{s}$ and $J_{t}$, where $s \in V\left(J_{s}\right)$ and $t \in V\left(J_{t}\right)$, and both $J_{s}^{\prime}:=J_{s}+w s$ and $J_{t}^{\prime}:=J_{t}+w t$ are 2-connected. Clearly $J_{s}^{\prime}$ and $J_{t}^{\prime}$ have plane representations so that $\partial J_{s}^{\prime}=w C s+w s$ and $\partial J_{t}^{\prime}=t C w+w t$. In $J_{s}^{\prime}$, we use Lemma (3.1) to find a $\partial J_{s}^{\prime}$-Tutte path $P_{s}$ from $w$ to $s$ and through $u v$. In $J_{t}^{\prime}$, we use Lemma (3.1) to find a $\partial J_{t}^{\prime}$-Tutte path $P_{t}^{\prime}$ from $w$ to $x$ and through $w t$, and let $P_{t}:=P_{t}^{\prime}-w$. It is easy to see that $P_{s} \cup P_{t}$ is a $t C s$-Tutte subgraph of $J$. By planarity, $u \in V\left(s P_{s} v\right)$.

It is easy to verify that $G^{\prime}:=(G-F)-V(J-\{s, t, w\}), H, Q, D, s, t, P^{\prime}, w$ (as $K, L, Q, Q^{\prime}, p, q, T, u$, respectively) satisfy the conditions of Lemma (3.3). By Lemma (3.3), we find a path $R \subseteq G^{\prime}-V\left(P^{\prime}\right)$ from $s$ to $t$ such that $R \cup P^{\prime}$ is a $Q$-Tutte subgraph of $G^{\prime}$ and every $P^{\prime}$-bridge of $H$ containing no edge of $D$ is an $\left(R \cup P^{\prime}\right)$-bridge of $G^{\prime}$. Since $\{a, b\} \subseteq V(Q \cap \partial G)$ and by planarity, $\{a, b\} \subseteq V(R)$. By (5) and since $S \cap V(D) \subseteq V\left(P^{\prime}\right), S \subseteq V\left(P^{\prime} \cup R\right)$.

Let $P:=P^{\prime} \cup R \cup P_{s} \cup P_{t}$. Then $P$ is a 1-way infinite path in $G$ from $x$ and through $u v$ such that $u \in V(x P v)$. It is easy to check that each non-trivial $P$-bridge of $G$ is one of the following: a $\left(R \cup P^{\prime}\right)$-bridge of $G^{\prime}$, or a $\left(P_{s} \cup P_{t}\right)$-bridge of $J$, or a subgraph of $H$ obtained from a $P^{\prime}$-bridge $B$ of $G$ by adding edges in $F$ between $S \cap V(B)$ and $V(B)-V\left(P^{\prime}\right)$, or a subgraph of $G$ obtained from a $P^{\prime}$-bridge $B$ of $H$ (with two attachments) by adding a vertex $s^{*} \in S$ and all edges in $F$ between $s^{*}$ and $V(B)-V\left(P^{\prime}\right)$. Hence, it is easy to see that $P$ is a 1 -way infinite $C$-Tutte path in $G$.

Case 2. $D \cap C$ is a non-trivial path.

Let $w, w^{\prime}$ denote the endvertices of $D \cap C$ such that $D \cap C=w^{\prime} C w$. Then $\left\{w^{\prime}, x\right\}$ is contained in an $(H \cup Q)$-bridge of $G-F$, and $\{w, u\}$ is contained in an $(H \cup Q)$-bridge of $G-F$. See Figure 6(c). In $H$, we use Lemma (4.1) to find a 1-way infinite $D$-Tutte path $P^{\prime}$ from $w$ and through $w^{\prime}$.

We claim that $S \cap V(D) \subseteq V\left(P^{\prime}\right)$. Suppose on the contrary that $s^{*} \in(S \cap V(D))-$ $V\left(P^{\prime}\right)$. Then $s^{*} \in V(B)-V\left(P^{\prime}\right)$ for some $P^{\prime}$-bridge $B$ of $H$. Since $P^{\prime}$ is a $D$-Tutte path of $H,\left|V\left(P^{\prime} \cap B\right)\right|=2$. Let $D_{w}$ and $D_{w}^{\prime}$ denote the infinite $w^{\prime} D w$-bridges of $D$ containing $w$ and $w^{\prime}$, respectively. (These are 1-way infinite paths.) By symmetry, assume that 
$s^{*} \in V\left(D_{w}^{\prime}\right)$. Then by planarity and since $\left\{w, w^{\prime}\right\} \subseteq V\left(P^{\prime}\right), V\left(B \cap P^{\prime}\right) \subseteq V\left(D_{w}^{\prime}\right)$. Note that $w \notin V\left(B \cap P^{\prime}\right)$. Hence $V\left(B \cap P^{\prime}\right)$ is a 2-cut of $H$ separating $s^{*}$ from $D_{w}$. Then either $V\left(B \cap P^{\prime}\right)$ separates the vertices in $S$ (when $|S \cap V(D)|=2$ ) or $V\left(B \cap P^{\prime}\right)$ separates $s^{*}$ from the component of $D \cap \partial N$ not containing $s^{*}$ (when $|S \cap V(D)|=1$ ), contradicting (5).

Next, we extend $P^{\prime}$ to the desired path $P$. Let $s \in V(Q)$ with $u Q s$ maximal such that $\{s, w\}$ is contained in an $(H \cup Q)$-bridge of $G-F$, and let $J_{s}$ denote the union of those $(H \cup Q)$-bridges of $G-F$ whose attachments are all contained in $V(u Q s) \cup\{w\}$. If $|V(w C s)|=2$ then let $P_{s}:=w C s$, and otherwise, we use Lemma (3.1) to find a $w C s$-Tutte path $P_{s}$ in $J_{s}+w s$ from $w$ to $s$ and through $u v$.

Let $t \in V(Q)$ with $t Q x$ maximal such that $\left\{t, w^{\prime}\right\}$ is contained in an $(H \cup Q)$-bridge of $G-F$, and let $J_{t}$ denote the union of those $(H \cup Q)$-bridges of $G-F$ whose attachments are all contained in $V(t Q x) \cup\left\{w^{\prime}\right\}$. If $t=x$ then let $P_{t}$ be the trivial path consisting of $x$, otherwise, we use Lemma (3.1) to find a $t C w^{\prime}$-Tutte path $P_{t}^{\prime}$ in $J_{t}+t w^{\prime}$ from $w^{\prime}$ to $x$ and through $t w^{\prime}$, and let $P_{t}:=P_{t}^{\prime}-w^{\prime}$.

It is easy to verify that $G^{\prime}:=(G-F)-V\left(\left(J_{s} \cup J_{t}\right)-\left\{s, t, w, w^{\prime}\right\}\right), H, Q, D, s, t, P^{\prime}, w$ (as $K, L, Q, Q^{\prime}, p, q, T, u$, respectively) satisfy the conditions of Lemma (3.3). By Lemma (3.3), we find a path $R \subseteq G^{\prime}-V\left(P^{\prime}\right)$ such that $R \cup P^{\prime}$ is a $Q$-Tutte subgraph of $G^{\prime}$ and every $P^{\prime}$-bridge of $H$ containing no edge of $D$ is an $\left(R \cup P^{\prime}\right)$-bridge of $G^{\prime}$. Since $\{a, b\} \subseteq V(Q \cap \partial G),\{a, b\} \subseteq V(R)$. By (5) and since $S \cap V(D) \subseteq V\left(P^{\prime}\right), S \subseteq V\left(P^{\prime} \cup R\right)$.

Let $P:=P^{\prime} \cup R \cup P_{s} \cup P_{t}$. Then $P$ is a 1-way infinite path in $G$ from $x$ and through $u v$ such that $u \in V(x P v)$. It is easy to verify that each non-trivial $P$-bridge of $G$ is one of the following: a $\left(R \cup P^{\prime}\right)$-bridge of $G^{\prime}$, or a $P_{s}$-bridge of $J_{s}^{\prime}$, or a $P_{t}^{\prime}$-bridge of $J_{t}^{\prime}$, or a subgraph of $G$ obtained from a $P^{\prime}$-bridge $B$ of $H$ by adding edges in $F$ between $S \cap V(B)$ and $V(B)-V\left(P^{\prime}\right)$, or a subgraph of $G$ obtained from a $P^{\prime}$-bridge $B$ of $H$ (with two attachments) by adding a vertex $s^{*} \in S$ and all edges in $F$ between $s^{*}$ and $V(B)-V\left(P^{\prime}\right)$. Hence, it is easy to see that $P$ is a 1 -way infinite $C$-Tutte path in $G$.

\section{One-way infinite paths}

In this section, we prove our main result about 1-way infinite Tutte paths from a specified vertex and through a specified edge. Such paths will be useful for proving the existence of 2-way infinite Tutte paths in 3-indivisible infinite plane graphs.

First, we state the following result, which is often referred to as König Lemma. It allows us to "construct" a 1-way infinite path from a sequence of finite paths.

(5.1) Lemma. Let $G$ be an infinite, locally finite graph, and let $x \in V(G)$. Suppose $\left\{P_{n}\right\}$ is an infinite sequence of finite paths from $x$ such that the length of $P_{n}$ increases. Then $\left\{P_{n}\right\}$ has a subsequence $\left\{P_{n_{k}}\right\}$ converging to a 1-way infinite path $P$ from $x$, that is, for every $v \in V(P), x P v=x P_{n_{k}} v$ for all sufficiently large $n_{k}$. 
In later proofs, we need to find a sequence of Tutte paths converging to a 1-way infinite Tutte path. For this reason, we recall the notion of forward paths. Let $N=\left(H_{1}, H_{2}, \cdots\right)$ be a sequence of finite subgraphs in a (finite or infinite) graph $G$. A path $P$ in $G$ is $N$ forward or $\left(H_{1}, H_{2}, \cdots\right)$-forward if, for $i \geq 1$ and for every $a, b, c \in V(P)$ with $a \in V(b P c)$, $\{b, c\} \subseteq V\left(H_{i}\right)$ implies that $a \notin V\left(H_{j}\right)$ for all $j \geq i+2$. Note that if, for each $i \geq 2$, $\bigcup_{j=1}^{i-1} H_{j}$ and $\bigcup_{j \geq i+1} H_{j}$ are contained in different components of $G-V\left(H_{i}\right)$, then " $P$ is $\left(H_{1}, H_{2}, \cdots\right)$-forward" means that if $P$ starts from $H_{1}$, then, after visiting $H_{i+2}, P$ never visits $H_{i}$ again.

Before we prove our main result, we need to prove two more lemmas.

(5.2) Lemma. Let $G$ be a 2-connected, 2-indivisible, infinite, plane graph with a radial net, let $C$ be a facial cycle of $G$ such that $G$ is $(4, C)$-connected, and let $x \in V(C)$. Then $G$ contains a 1-way infinite $C$-Tutte path $P$ from $x$.

Proof. By Lemma (2.2), we may work with a nice embedding of $G$ in which $C$ is a facial cycle. First, we construct an infinite sequence $\mathcal{G}=\left(\left(G_{i}, C_{i}, x_{i}\right): i \geq 1\right)$. Let $G_{1}=G, C_{1}=C$, and $x_{1}=x$. Suppose for some $i \geq 1$, we have constructed a 2connected, infinite, plane graph $G_{i} \subseteq G$ with a radial net, a facial cycle $C_{i}$ of $G_{i}$, and a vertex $x_{i} \in V\left(C_{i}\right)$. Let $G_{i+1}$ denote the unique infinite block of $G_{i}-V\left(C_{i}\right)$ and let $C_{i+1}$ denote the facial cycle of $G_{i+1}$ for which $C_{i} \subseteq I_{G_{i}}\left(C_{i+1}\right)$. (Both $G_{i+1}$ and $C_{i+1}$ exist, since $G_{i}$ has a radial net.)

It is easy to see that the conditions of Lemma (3.7) are satisfied, with $G_{i}, C_{i}, x_{i}, G_{i+1}, C_{i+1}$ as $G, C, x, G^{\prime}, C^{\prime}$, respectively. By Lemma (3.7), we have the following.

(1) There exists some $x_{i+1} \in V\left(C_{i+1}\right)$ such that, for any (finite or infinite) subgraph $X$ of $G_{i}$ containing $I_{G_{i}}\left(C_{i+1}\right)$ and for any (finite or 1-way infinite) $C_{i+1}$-Tutte path $P_{i+1}$ from $x_{i+1}$ in $X^{\prime}:=X \cap G_{i+1}$, there is a $C_{i}$-Tutte path $P_{i}$ from $x_{i}$ in $G_{i}$ such that $P_{i+1} \subseteq P_{i}, P_{i}-V\left(P_{i+1}-x_{i+1}\right)$ is a path from $x_{i}$ to $x_{i+1}$, and for any $z \in V\left(P_{i}\right)-V\left(P_{i+1}\right)$, either $z \notin V\left(X^{\prime}\right)$ or $z \in V(Z)-V\left(P_{i+1)}\right.$ for some $P_{i+1}$-bridge $Z$ of $X^{\prime}$ containing an edge of $C_{i+1}$.

Recall that $N=\left(C_{1}, C_{2}, \cdots\right)$ is a radial net in $G$. Let $H_{i}=\left(I_{G}\left(C_{i+1}\right)-V\left(C_{i+1}\right)\right)-$ $V\left(I_{G}\left(C_{i}\right)-V\left(C_{i}\right)\right)$, and let $G_{n, i}=G_{i} \cap I_{G}\left(C_{n}\right)$ (for $n \geq i \geq 1$ ). By definition, $G_{n, i}=$ $I_{G}\left(C_{n}\right)-V\left(I_{G}\left(C_{i}\right)-V\left(C_{i}\right)\right)$, and $H_{1}, H_{2}, \ldots$ are pairwise vertex disjoint. Next we show that

(2) $G_{n, i}$ contains a $C_{i}$-Tutte path $P_{n, i}$ between $x_{i}$ and a vertex of $C_{n}$ such that $P_{n, i}$ is $\left(H_{1}, H_{2}, \cdots\right)$-forward in $G$. 
We use induction on $n-i$. If $n-i=0$, then $G_{n, i}=C_{n}=C_{i}$. In this case, let $P_{n, i}$ be a path in $C_{n}$ between $x_{i}$ and an arbitrary vertex of $C_{n}-x_{i}$. Then $P_{n, i}$ is a $C_{i}$-Tutte path in $G_{n, i}$ (because $G_{n, i}=C_{n}$ has only one $P_{n, i}$-bridge which has just two attachments) and $P_{n, i}$ is $\left(H_{1}, H_{2}, \cdots\right)$-forward (because $\left.P_{n, i} \subseteq C_{n} \subseteq H_{n}\right)$.

Now assume that $n-i \geq 1$ and $G_{n, i+1}$ contains a $C_{i+1}$-Tutte path $P_{n, i+1}$ between $x_{i+1}$ and a vertex of $C_{n}$ such that $P_{n, i+1}$ is $\left(H_{1}, H_{2}, \cdots\right)$-forward in $G$. By (1) above (with $\left.X=G_{n, i}\right) G_{n, i}$ contains a $C_{i}$-Tutte path $P_{n, i}$ from $x_{i}$ such that (a) $P_{n, i+1} \subseteq P_{n, i}$, (b) $P_{n, i}-V\left(P_{n, i+1}-x_{i+1}\right)$ is a path between $x_{i}$ and $x_{i+1}$, and (c) for any $z \in V\left(P_{n, i}\right)-$ $V\left(P_{n, i+1}\right)$, either $z \notin V\left(G_{n, i+1}\right)$ or $z \in V(Z)-V\left(P_{n, i+1}\right)$ for some $P_{n, i+1}$-bridge $Z$ of $G_{n, i+1}$ containing an edge of $C_{i+1}$. By (b), $P_{n, i}$ is between $x_{i}$ and a vertex of $C_{n}$. By (c) and since every $P_{n, i+1}$-bridge of $G_{n, i+1}$ containing an edge of $C_{i+1}$ has just two attachments, $\left(P_{n, i}-V\left(P_{n, i+1}-x_{i+1}\right)\right) \cap C_{i+2}=\emptyset$. Hence, $P_{n, i}-V\left(P_{n, i+1}-x_{i+1}\right) \subseteq$ $H_{i} \cup H_{i+1}$.

To show that $P_{n, i}$ is $\left(H_{1}, H_{2}, \cdots\right)$-forward in $G$, let $a, b, c \in V\left(P_{n, i}\right)$ such that $a \in$ $V\left(b P_{n, i} c\right)$, and $b, c \in V\left(H_{k}\right)$. We need to show that $a \notin V\left(H_{j}\right)$ for all $j \geq k+2$. First, assume that $b, c \in V\left(P_{n, i}\right)-V\left(P_{n, i+1}-x_{i+1}\right)$. Then $b P_{n, i} c \subseteq P_{n, i}-V\left(P_{n, i+1}-x_{i+1}\right) \subseteq$ $H_{i} \cup H_{i+1}$. Hence, $H_{k}=H_{i}$ or $H_{k}=H_{i+1}$. Since $a \in V\left(b P_{n, i} c\right), a \in V\left(H_{i}\right) \cup V\left(H_{i+1}\right)$, and so, $a \notin V\left(H_{j}\right)$ for all $j \geq k+2 \geq i+2$. Now assume that $b, c \in V\left(P_{n, i+1}\right)$. Then $a \notin V\left(H_{j}\right)$ for all $j \geq k+2$ because $P_{n, i+1}$ is $\left(H_{1}, H_{2}, \cdots\right)$-forward in $G$. Finally, assume by symmetry that $b \in V\left(P_{n, i}\right)-V\left(P_{n, i+1}\right)$ and $c \in V\left(P_{n, i+1}-x_{i+1}\right)$. Then $b \in$ $V\left(H_{i}\right) \cup V\left(H_{i+1}\right)$ and $c \notin V\left(H_{i}\right)$. Since $b, c \in V\left(H_{k}\right), H_{k}=H_{i+1}$, and so, $x_{i+1} \in V\left(H_{k}\right)$. Since $a \in V\left(x_{i+1} P_{n, i+1} c\right)$ and $\left\{x_{i+1}, c\right\} \subseteq V\left(H_{k}\right), a \notin V\left(H_{j}\right)$ for all $j \geq k+2$ (because $P_{n, i+1}$ is $\left(H_{1}, H_{2}, \cdots\right)$-forward in $\left.G\right)$. Hence, $P_{n, i}$ is $\left(H_{1}, H_{2}, \cdots\right)$-forward in $G$. This completes the proof of $(2)$.

By (2), $P_{n}:=P_{n, 1}$ is a $C$-Tutte path in $G_{n, 1}=I\left(C_{n}\right)$ between $x$ and a vertex of $C_{n}$, and $P_{n}$ is $\left(H_{1}, H_{2}, \cdots\right)$-forward in $G$. By Lemma (5.1), there is subsequence $\left\{P_{n_{k}}\right\}$ of $\left\{P_{n}\right\}$ converging to a 1-way infinite path $P$ from $x$ in $G$. We claim that

(3) for any $P$-bridge $B$ of $G, B$ is a $P_{n_{k}}$-bridge of $I_{G}\left(C_{n_{k}}\right)$ for all sufficiently large $n_{k}$.

First, we see that $B$ must be finite. For otherwise, since $G$ is locally finite (because $G$ has a radial net), $B$ contains a 1-way infinite path. Thus a finite subpath $Q$ of that 1-way infinite path must intersect $C_{i}, \ell \leq i \leq \ell+3$, for some large $\ell$. Now $Q \subseteq I_{G}\left(C_{j}\right)$ for all sufficiently large $j$. So $Q$ is contained in some $P_{n_{k}}$-bridge of $I_{G}\left(C_{n_{k}}\right)$ for all sufficiently large $n_{k}$. Since $Q$ intersects at least four consecutive $C_{i}$ 's, such a $P_{n_{k}}$-bridge of $I_{G}\left(C_{n_{k}}\right)$ has at least four attachments, a contradiction. Now that $B$ is finite, $B \subseteq I_{G}\left(C_{i}\right)$ for all sufficiently large $i$. Therefore, $B$ is a $P_{n_{k}}$-bridge of $I_{G}\left(C_{n_{k}}\right)$ for all sufficiently large $n_{k}$.

By (3) and since each $P_{n_{k}}$ is a $C$-Tutte path of $I_{G}\left(C_{n_{k}}\right), P$ is a 1-way infinite $C$-Tutte path from $x$ in $G$. 
The next lemma will serve as part of the induction basis in the proof of our main result.

(5.3) Lemma. Let $G$ be a 2-connected, 2-indivisible, infinite, plane graph, and $C$ be a facial cycle of $G$ such that $G$ is $(4, C)$-connected. Then, for every $x \in V(C), G$ contains a 1-way infinite $C$-Tutte path from $x$.

Proof. If $G$ has a radial net, then Lemma (5.3) follows from Lemma (5.2). Now assume that $G$ has no radial net. Let $S$ denote the set of vertices of infinite degree in $G$. Then there is a set $F \subseteq E(G)$ as in Theorem (2.1) such that $G-F$ has a ladder net $N$ satisfying the conclusions of Theorem (2.1).

Thus, let $n$ denote the maximum number for which there are vertex disjoint cycle $C_{0}, C_{1}, \ldots, C_{n}$ in $G$ such that $C_{0}=C$ and $I\left(C_{0}\right) \subseteq I\left(C_{1}\right) \subseteq \ldots \subseteq I\left(C_{n}\right)$. Then $C_{n} \cap \partial G \neq$ $\emptyset$. We will apply induction on $n$.

Suppose $n=0$. Since $G$ is $(4, C)$-connectd, we can pick $u v$ from $E(C)$ so that $v \neq x$, the path $Q$ of $C-v$ between $x$ and $u$ intersects $\partial G$, and $v$ is in the infinite component of $G-V(Q)$. Hence Lemma (5.3) follows from Lemma (4.2).

So assume that $n \geq 1$, and consider $G-V(C)$. Let $H$ denote the unique infinite block of $G-V(C)$ (which exists since $G$ is 2-indivisible and (4,C)-connected) and let $C^{\prime}$ denote the cycle bounding the face of $H$ containing $C$. By Lemma (3.7), there is some $x^{\prime} \in V\left(C^{\prime}\right)$ such that, for any 1-way infinite $C^{\prime}$-Tutte path $P^{\prime}$ from $x^{\prime}$ in $H$, there is a 1way infinite $C$-Tutte path $P$ from $x$ in $G$ with $P^{\prime} \subseteq P$. Note that if $D_{0}=C^{\prime}, D_{1}, \ldots, D_{k}$ are disjoint cycles of $H$ with $I_{H}\left(D_{0}\right) \subseteq I_{H}\left(D_{1}\right) \subseteq \ldots \subseteq I_{H}\left(D_{k}\right)$, then $k<n$ by the maximality of $n$. So by induction, there is a 1-way infinite $C^{\prime}$-Tutte path $P^{\prime}$ in $H$ from $x^{\prime}$. Hence, $G$ has a 1 -way infinite $C$-Tutte path from $x$.

Proof of Theorem (1.2). It follows from Lemma (2.2) that we may work with a nice embedding of $G$ in which $C$ is a facial cycle. Recall that $\partial G=\emptyset$ if and only if $G$ has a radial net.

First, we construct a sequence $\mathcal{G}=\left\{\left(G_{i}, C_{i}, Q_{i}, x_{i}, u_{i} v_{i}\right): i=0,1,2, \cdots\right\}$. Let $G_{0}=$ $G, C_{0}=C, Q_{0}=Q, x_{0}=x, u_{0}=u$, and $v_{0}=v$.

If $Q_{0} \cap \partial G_{0} \neq \emptyset$, then we stop this process.

Suppose for some $i \geq 0$, we have constructed $\left(G_{j}, C_{j}, Q_{j}, x_{j}, u_{j} v_{j}\right), 0 \leq j \leq i$, such that for every $0 \leq j \leq i, G_{j} \subseteq G$ is a 2-connected, 2-indivisible, infinite, plane graph, $C_{j}$ is a facial cycle of $G_{j}, G_{j}$ is $\left(4, C_{j}\right)$-connected, $x_{j} \in V\left(C_{j}\right)$ and $u_{j} v_{j} \in E\left(C_{j}\right)$ with $v_{j} \neq x_{j}, Q_{j}$ is the subpath of $C_{j}-v_{j}$ between $x_{j}$ and $u_{j}, v_{j}$ is in the infinite component of $G_{j}-V\left(Q_{j}\right)$, and for all $0 \leq j<i, I_{G}\left(C_{j}\right) \subseteq I_{G}\left(C_{j+1}\right)$, and any $\left(G_{j+1} \cup C_{j}\right)$-bridge of $G_{j}$ has at most one attachment on $C_{j+1}$.

If $Q_{i} \cap \partial G \neq \emptyset$, then we stop this process.

Now assume $Q_{i} \cap \partial G=\emptyset$. Then since $G_{i}$ is 2-indivisible and $\left(4, C_{i}\right)$-connected, $G_{i}-V\left(Q_{i}\right)$ has a unique infinite block, say $H_{i}$, which has a facial cycle $C_{i}^{\prime}$ bounding the 
face of $H_{i}$ containing $C_{i}$. By planarity there are two possibilities: $\left|V\left(C_{i}\right) \cap V\left(C_{i}^{\prime}\right)\right| \leq 1$ or $C_{i} \cap C_{i}^{\prime}$ is a nontrivial path. (See Figures 3 and 4 for an illustration.) Recall that $v_{i}$ and $H_{i}$ are contained in the unique infinite component of $G_{i}-V\left(Q_{i}\right)$.

(i) If $\left|V\left(C_{i}\right) \cap V\left(C_{i}^{\prime}\right)\right| \leq 1$, then by Lemma (3.5) (with $G_{i}, C_{i}, Q_{i}, H_{i}, C_{i}^{\prime}, x_{i}, u_{i}, v_{i}$ as $G, C, Q, G^{\prime}, C^{\prime}, x, u, v$, respectively), there exists $x_{i}^{\prime} \in V\left(C_{i}^{\prime}\right)$ such that, for any $C_{i}^{\prime}$-Tutte path $P_{i}^{\prime}$ from $x_{i}^{\prime}$ in $H_{i}$ with $\left|V\left(P_{i}^{\prime}\right) \cap V\left(C_{i}^{\prime}\right)\right| \geq 2$ there is a $C_{i}$-Tutte path $P_{i}$ from $x_{i}$ through $u_{i} v_{i}$ in $G_{i}$ with the following properties: (a) $P_{i}^{\prime} \subseteq P_{i}$, (b) $u_{i} \in V\left(x_{i} P_{i} v_{i}\right)$ and $P_{i}-V\left(P_{i}^{\prime}-x_{i}^{\prime}\right)$ is a path from $x_{i}$ to $x_{i}^{\prime}$, and (c) for any $z \in V\left(P_{i}\right)-V\left(P_{i}^{\prime}\right)$, either $z \notin V\left(H_{i}\right)$ or $z \in V(Z)-V\left(P_{i}^{\prime}\right)$ for some $P_{i}^{\prime}$-bridge $Z$ of $H_{i}$.

(ii) If $C_{i} \cap C_{i}^{\prime}$ is a nontrivial path then by Lemma (3.6) (with $G_{i}, C_{i}, Q_{i}, H_{i}, C_{i}^{\prime}, x_{i}, u_{i}, v_{i}$ as $G, C, Q, G^{\prime}, C^{\prime}, x, u, v$, respectively), there exist $x_{i}^{\prime} \in V\left(C_{i}\right) \cap V\left(C_{i}^{\prime}\right)$ and $u_{i}^{\prime} v_{i}^{\prime} \in E\left(C_{i}^{\prime}\right)$ such that $x_{i}^{\prime}$ and $u_{i}^{\prime}$ are the endvertices of $C_{i} \cap C_{i}^{\prime}, v_{i}^{\prime}$ is in the infinite component of the graph obtained from $H_{i}$ by deleting the path $Q_{i}^{\prime}$ in $C_{i}^{\prime}-v_{i}^{\prime}$ between $x_{i}^{\prime}$ and $u_{i}^{\prime}$, and, for any subgraph $X$ of $G_{i}$ containing $I_{G}\left(C_{i}^{\prime}\right)$ and for any $C_{i}^{\prime}$-Tutte path $P_{i}^{\prime}$ in $X^{\prime}:=X \cap H_{i}$ from $x_{i}^{\prime}$ and through $u_{i}^{\prime} v_{i}^{\prime}$, there is a $C_{i}$-Tutte path from $x_{i}$ through $u_{i} v_{i}$ in $X$ with the following properties: (a) $P_{i}^{\prime} \subseteq P_{i}$, (b) $u_{i} \in V\left(x_{i} P_{i} v_{i}\right)$ and $P_{i}-V\left(P_{i}^{\prime}-x_{i}^{\prime}\right)$ is a path from $x_{i}$ to $x_{i}^{\prime}$, and (c) for any $z \in V\left(P_{i}\right)-V\left(P_{i}^{\prime}\right)$, either $z \notin V\left(H_{i}\right)$ or $z \in V(Z)-V\left(P_{i}^{\prime}\right)$ for some $P_{i}^{\prime}$-bridge $Z$ of $X^{\prime}$.

If (i) occurs, we stop this process.

Now assume (ii) occurs. Let $G_{i+1}=G_{i}^{\prime}, C_{i+1}=C_{i}^{\prime}, Q_{i+1}=Q_{i}^{\prime}, x_{i+1}=x_{i}^{\prime}, u_{i+1}=$ $u_{i}^{\prime}$, and $v_{i+1}=v_{i}^{\prime}$. Since $x_{i+1}$ and $u_{i+1}$ are endvertices of $C_{i} \cap C_{i}^{\prime}$, we have $Q_{i+1}=$ $C_{i} \cap C_{i+1}$ or $Q_{i+1}=C_{i+1}-\left(V\left(C_{i} \cap C_{i+1}\right)-\left\{x_{i+1}, u_{i+1}\right\}\right)$. Note that $G_{i+1}$ is a 2connected, 2-indivisible, infinite, plane graph, $C_{i+1}$ is a facial cycle of $G_{i+1}, G_{i+1}$ is (4, $\left.C_{i+1}\right)$-connected, $x_{i+1} \in V\left(C_{i+1}\right)$ and $u_{i+1} v_{i+1} \in E\left(C_{i+1}\right)$ with $v_{i+1} \neq x_{i+1}, Q_{i+1}$ is the subpath of $C_{i+1}-v_{i+1}$ between $x_{i+1}$ and $u_{i+1}, v_{i+1}$ is in the infinite component of $G_{i+1}-V\left(Q_{i+1}\right), I_{G}\left(C_{i}\right) \subseteq I_{G}\left(C_{i+1}\right)$, and any $\left(G_{i+1} \cup C_{i}\right)$-bridge of $G_{i}$ has at most one attachment on $C_{i+1}$.

(1) We may assume that $\mathcal{G}$ is an infinite sequence.

Otherwise, suppose that $\mathcal{G}=\left\{\left(G_{i}, C_{i}, Q_{i}, x_{i}, u_{i} v_{i}\right): i=0, \cdots, n\right\}$. Then by the above construction of $\mathcal{G}$, either $Q_{n} \cap \partial G \neq \emptyset$ or $\left|V\left(C_{n} \cap C_{n}^{\prime}\right)\right| \leq 1$. We will apply induction on $n$.

Suppose $n=0$. If $Q_{0} \cap \partial G \neq \emptyset$, then the result follows from Lemma (4.2). So assume that $Q_{0} \cap \partial G=\emptyset$. Then $\left|V\left(C_{0} \cap C_{0}^{\prime}\right)\right| \leq 1$. By Lemma (5.2), $H_{0}$ has a 1-way infinite $C_{0}^{\prime}$-Tutte path from $x_{0}^{\prime}$. By (i) in the construction of $\mathcal{G}$, we see that $G_{0}=G$ has a 1-way infinite $C$-Tutte path $P$ from $x$ through $u v$ such that $u \in V(x P v)$.

Now assume that $n \geq 1$. Then by the above construction of $\mathcal{G}, Q_{0} \cap \partial G=\emptyset$ and $C_{0} \cap C_{0}^{\prime}$ is a nontrivial path. Therefore, we may apply induction to the sequence $\mathcal{G}_{1}=\left\{\left(G_{i}, C_{i}, Q_{i}, x_{i}, u_{i} v_{i}\right): i=1, \cdots, n\right\}$, and conclude that $G_{1}$ has a 1 -way infinite 
$C_{1}$-Tutte path $P_{1}$ from $x_{1}$ through $u_{1} v_{1}$. By (ii) in the construction of $\mathcal{G}$, we see that $G_{0}=G$ has a 1 -way infinite $C$-Tutte path $P$ from $x$ through $u v$ such that $u \in V(x P v)$. This proves (1).

By (1), $Q_{j} \cap \partial G \neq \emptyset$ and $C_{j} \cap C_{j-1}$ is a non-trivial path, for all $j \geq 1$. Hence for all $j \geq 1, Q_{j}=C_{j} \cap C_{j-1}$ or $Q_{j}=C_{j-1}-V\left(\left(C_{j} \cap C_{j-1}\right)-\left\{x_{j}, u_{j}\right\}\right)$. Also from the construction of $\mathcal{G}$, we have $I_{G}\left(C_{j-1}\right) \subseteq I_{G}\left(C_{j}\right)$, for all $j \geq 1$. We will show that there is a subsequence $\left(C_{i_{1}}, C_{i_{2}}, \ldots\right)$ of $\left(C_{1}, C_{2}, \ldots\right)$ such that $C_{i_{1}}=C_{0}$ and $C_{i_{j}} \cap C_{i_{k}}=\emptyset$ for all $i_{j} \neq i_{k}$. This sequence will be used to define forward Tutte paths.

(2) We claim that, for any given $i \geq 1$, there is some $\ell_{i}>i$ such that $C_{\ell_{i}} \cap C_{i}=\emptyset$.

Suppose that $C_{j} \cap C_{i} \neq \emptyset$ for all $j>i$. First we show that $Q_{j}=C_{j}-V\left(\left(C_{j} \cap C_{j-1}\right)-\right.$ $\left.\left\{x_{j}, u_{j}\right\}\right)$ for all $j>i$. For otherwise, assume that $Q_{k}=C_{k} \cap C_{k-1}$ for some $k>i$. Then $C_{k+1} \cap C_{k-1}=\emptyset$ because $C_{k+1}=C_{k}^{\prime} \subseteq G_{k}-V\left(Q_{k}\right)=G_{k}-V\left(C_{k} \cap C_{k-1}\right)=G_{k}-V\left(C_{k-1}\right)$ (since $\left.\left(C_{k-1}-V\left(C_{k} \cap C_{k-1}\right)\right) \cap G_{k}=\emptyset\right)$. Since $I_{G}\left(C_{j}\right) \subseteq I_{G}\left(C_{j+1}\right)$ for all $j \geq 1$, $C_{k+1} \cap C_{k} \subseteq C_{k+1} \cap C_{k-1}=\emptyset$, a contradiction.

Hence, for all $j \geq i, C_{j+1} \cap C_{j} \subseteq C_{j}-V\left(Q_{j}\right)=\left(C_{j} \cap C_{j-1}\right)-\left\{x_{j}, u_{j}\right\} \neq C_{j} \cap C_{j-1}$. That is, for all $j \geq i, C_{j+1} \cap C_{j}$ is a proper subgraph of $C_{j} \cap C_{j-1}$. But this is impossible because $C_{i} \cap C_{i+1}$ is finite. Hence we have (2).

By (2), let $N=\left(C_{i_{1}}, C_{i_{2}}, \cdots\right)$ be a subsequence of $\left(C_{0}, C_{1}, \cdots\right)$ such that $C_{i_{1}}=C_{0}$ and $C_{i_{k}} \cap C_{i_{k+1}}=\emptyset$ for all $k \geq 0$. Then $N$ is a radial net in $G$. Let $H_{k}=\left(I\left(C_{i_{k+1}}\right)-\right.$ $\left.V\left(C_{i_{k+1}}\right)\right)-V\left(I\left(C_{i_{k}}\right)-V\left(C_{i_{k}}\right)\right)$. For $1 \leq i \leq n$, let $G_{n, i}=G_{i} \cap I\left(C_{n}\right)$. Next we show that

(3) $G_{n, i}$ contains a $C_{i}$-Tutte path $P_{n, i}$ between $x_{i}$ and a vertex of $C_{n}$ such that $u_{i} v_{i} \in$ $E\left(P_{n, i}\right), u_{i} \in V\left(x_{i} P_{n, i} v_{i}\right)$, and $P_{n, i}$ is $\left(H_{1}, H_{2}, \cdots\right)$-forward in $G$.

We use induction on $n-i$. If $n=i$, then $G_{n, i}=C_{i}=C_{n}$. In this case, let $f$ be the edge of $C_{i}$ incident with $x_{i}$ such that $f$ is contained in the path of $C-u_{i}$ between $x_{i}$ and $v_{i}$, and let $P_{n, i}=C_{i}-f$. It is easy to see that $P_{n, i}$ is a $C_{i}$-Tutte path between $x_{i}$ and a vertex of $C_{n}$ such that $u_{i} v_{i} \in E\left(P_{i}\right), u_{i} \in V\left(x_{i} P_{n, i} v_{i}\right)$, and $P_{n, i}$ is $\left(H_{1}, H_{2}, \cdots\right)$-forward in $G$ (because $P_{n, i} \subseteq C_{n} \subseteq H_{k}$ for some $k$ ).

Now assume that $n>i$ and $G_{n, i+1}$ contains a $C_{i+1}$-Tutte path $P_{n, i+1}$ between $x_{i+1}$ and a vertex of $C_{n}$ such that $u_{i+1} v_{i+1} \in E\left(P_{i+1}\right), u_{i+1} \in V\left(x_{i+1} P_{n, i+1} v_{i+1}\right)$, and $P_{n, i+1}$ is $\left(H_{1}, H_{2}, \cdots\right.$ )-forward in $G$. By (ii) in the construction of $\mathcal{G}$ (with $X=G_{n, i}$ ), $G_{n, i}$ has a $C_{i}$-Tutte path $P_{n, i}$ from $x_{i}$ and through $u_{i} v_{i}$ such that (a) $P_{n, i+1} \subseteq P_{n, i}$ and $u_{i} \in V\left(x_{i} P_{n, i} v_{i}\right)$, (b) $P_{n, i}-V\left(P_{n, i+1}-x_{i+1}\right)$ is a path between $x_{i}$ and $x_{i+1}$, and (c) for any $z \in V\left(P_{n, i}\right)-V\left(P_{n, i+1}\right)$, either $z \notin V\left(G_{n, i+1}\right)$ or $z \in V(Z)-V\left(P_{n, i+1}\right)$ for some $P_{n, i+1}$-bridge $Z$ of $G_{n, i+1}$ containing an edge of $C_{i+1}$. 
It remains to show that $P_{n, i}$ is $\left(H_{1}, H_{2}, \cdots\right)$-forward in $G$. Note that $C_{i} \subseteq H_{l}$ for some positive integer $l$. Then from the construction of $\mathcal{G}$ (only (ii) applies since $\mathcal{G}$ is infinite), we have $x_{i+1} \in V\left(H_{l}\right)$ because $x_{i+1} \in V\left(C_{i}\right)$. Also, by (c) above, $P_{n, i}-$ $V\left(P_{n, i+1}-x_{i+1}\right) \subseteq H_{l} \cup H_{l+1}$. Let $a, b, c \in V\left(P_{n, i}\right)$ such that $a \in V\left(b P_{n, i} c\right)$, and suppose that $\{b, c\} \subseteq V\left(H_{k}\right)$. We need to show that $a \notin V\left(H_{j}\right)$ for all $j \geq k+2$. If $\{b, c\} \subseteq V\left(P_{n, i+1}\right)$, then $a \notin V\left(H_{j}\right)$ for all $j \geq k+2$ because $P_{n, i+1}$ is $\left(H_{1}, H_{2}, \cdots\right)$ forward in $G$. Now assume that $\{b, c\} \subseteq V\left(P_{n, i}\right)-V\left(P_{n, i+1}-x_{i+1}\right)$. Then $a \in V\left(b P_{n, i} c\right) \subseteq$ $V\left(P_{n, i}\right)-V\left(P_{n, i+1}-x_{i+1}\right) \subseteq V\left(H_{l} \cup H_{l+1}\right)$. Hence, either $H_{k}=H_{l}$ or $H_{k}=H_{l+1}$, and so, $a \notin V\left(H_{j}\right)$ for any $j \geq k+2 \geq l+2$. Finally, assume by symmetry that $b \notin V\left(P_{n, i+1}\right)$ and $c \in V\left(P_{n, i+1}-x_{i+1}\right)$. Then $b \in V\left(H_{l}\right) \cup V\left(H_{l+1}\right)$, and hence, either $H_{k}=H_{l}$ or $H_{k}=H_{l+1}$. We may assume that $a \in V\left(P_{n, i+1}\right)$; otherwise, $\left.a \in V H_{l}\right) \cup V\left(H_{l+1}\right)$, and hence, $a \notin V\left(H_{j}\right)$ for all $j \geq k+2 \geq l+2$. If $H_{k}=H_{l}$, then $a \notin V\left(H_{j}\right)$ for all $j \geq k+2$, because $a \in V\left(x_{i+1} P_{n, i+1} c\right),\left\{x_{i+1}, c\right\} \subseteq V\left(H_{k}\right)$, and $P_{n, i+1}$ is $\left(H_{1}, H_{2}, \cdots\right)$-forward in $G$. So assume that $H_{k}=H_{l+1}$. Now suppose that $a \in V\left(H_{r}\right)$ for some $r \geq k+2$. Since $x_{i+1} \in V\left(H_{l}\right)$, there is some $x^{\prime} \in V\left(x_{i+1} P_{n, i+1} a\right) \cap V\left(H_{k}\right)$. Hence $\left\{x^{\prime}, c\right\} \subseteq V\left(H_{k}\right)$ and $a \in V\left(x^{\prime} P_{n, i+1} c\right)$. Since $P_{n, i+1}$ is $\left(H_{1}, H_{2}, \cdots\right)$-forward in $G, a \notin V\left(H_{j}\right)$ for all $j \geq k+2$, contradicting the assumption that $a \in V\left(H_{r}\right)$. Hence, $P_{n, i}$ is $\left(H_{1}, H_{2}, \cdots\right)$-forward in $G$.

Let $P_{n}=P_{n, 1}$. Then $P_{n}$ is a $C$-Tutte path in $I\left(C_{n}\right)$ between $x$ and a vertex of $C_{n}$ and through $u v$ such that $u \in V\left(x P_{n} v\right)$, and $P_{n}$ is $\left(H_{1}, H_{2}, \cdots\right)$-forward in $G$. By Lemma (5.1), $\left\{P_{n}\right\}$ has a subsequence $\left\{P_{n_{k}}\right\}$ converging to a 1-way infinite path $P$ from $x$ and through $u v$. Note that $u \in V(x P v)$ (since $u \in V\left(x P_{n_{k}} v\right)$ for all $k \geq 1$ ). By a similar argument as for (3) in the proof of (5.2), we have

(4) for any $P$-bridge $B$ of $G, B$ is a $P_{n_{k}}$-bridge of $I\left(C_{n_{k}}\right)$ for all sufficiently large $n_{k}$.

By (4) and since each $P_{n_{k}}$ is a $C$-Tutte path of $I\left(C_{n_{k}}\right), P$ is a 1-way infinite $C$-Tutte path in $G$ from $x$ and through $u v$ such that $u \in V(x P v)$.

It is easy to see that Theorem (1.1) follows from Theorem (1.2).

\section{References}

[1] N. Dean, R. Thomas and X. Yu, Spanning paths in infinite planar graphs, J. Graph Theory, 23 (1996) 163-174.

[2] C. St. J. A. Nash-Williams, Hamiltonian lines in infinite graphs with few vertices of small valency, Aequationes Math. 7 (1971) 59-81.

[3] C. St. J. A. Nash-Williams, Unexplored and semi-explored territories in graph theory in New Directions in Graph Theory (ed. F. Harary), Academic Press (1973) 149-186. 
[4] R. Thomas and X. Yu, 4-Connected projective-planar graphs are hamiltonian, $J$. Combinat. Theory Ser. B 62 (1994) 114-132.

[5] C. Thomassen, Infinite graphs in Selected Topics in Graph Theory Vol. 2, Academic Press (1983) 129-160.

[6] C. Thomassen, A theorem on paths in planar graphs, J. Graph Theory, 7 (1983) 169-176.

[7] T. Tutte, A theorem on planar graphs, Trans. Amer. Math. Soc. 82 (1956) 99-116.

[8] H. Whitney, A theorem on graphs. Ann. of Math. 32 (1931) 378-390.

[9] X. Yu, Infinite paths in planar graphs I, graphs with radial nets, J. Graph Theory, to appear.

[10] X. Yu, Infinite paths in planar graphs II, structures and ladder nets, Submitted. 